\title{
Single particle-resolution fluorescence microscopy of nanoplastics
}

\section{Content Type: Letter}

\author{
Authors \\ Brian Nguyen $\uparrow$, Nathalie Tufenkji†
}

\section{Affiliations \\ $\dagger$ Department of Chemical Engineering, McGill University, 3610 University street, Montreal, Quebec H3A 0C5, Canada}

\begin{abstract}
Understanding of nanoplastic prevalence and toxicology is limited by imaging challenges resulting from their small size. Fluorescence microscopy is widely applied to track and identify microplastics in laboratory studies and environmental samples. However, conventional fluorescence microscopy, due to diffraction, lacks the resolution to precisely localize nanoplastics in tissues, distinguish them from free dye, or quantify them in environmental samples. To address these limitations, we developed techniques to label nanoplastics for imaging with Stimulated Emission Depletion (STED) microscopy to achieve resolution at an order of magnitude superior to conventional fluorescence microscopy. These techniques include (1) passive sorption; (2) swell incorporation; and (3) covalent coupling of STED-compatible fluorescence dyes to nanoplastics. We demonstrate that our labeling techniques, combined with STED microscopy, can be used to resolve nanoplastics of different shapes and compositions as small as $50 \mathrm{~nm}$. The longevity of the dye labeling is demonstrated in different media and conditions of biological and environmental relevance. We also test STED imaging of nanoplastics in exposure experiments with the model worm $C$. elegans. These techniques will allow more precise localization and quantification of nanoplastics in complex matrices.
\end{abstract}

\section{Main}

The visibility of plastic pollution and the potential effects on biota have garnered considerable attention. The vast majority of plastics produced ends up in landfills or the environment ${ }^{1}$. Once in the environment, plastic can fragment into smaller pieces known as secondary plastics, via mechanical, thermal, or other mechanisms of degradation ${ }^{2-7}$. As a result, the smallest size fractions of plastic pollution often dominate particle counts in the environment ${ }^{8,9}$. Despite their potential prevalence, little is known about the toxicological effects and mechanisms of the smallest particles (i.e., nanoplastics) in part due to the difficulty of visualizing them in wet matrices including biological tissues.

Fluorescence microscopy allows sensitive and quantitative imaging with specific labeling of particles and biological structures. Consequently, fluorescence imaging has been widely applied to localize labeled microplastic particles used in exposure studies to track uptake and potential translocalization in live organisms ${ }^{11-13}$. Typically, microplastics are large enough to be individually resolved with light microscopy ${ }^{11-13}$, allowing localization of fluorescently-labeled microplastics even if there is significant background signal from autofluorescence or dye leaching ${ }^{14,15}$. However, because of the optical $200 \mathrm{~nm}$ diffraction limit on resolution, nanoplastics are typically too small to be individually resolved with fluorescence microscopy techniques currently applied in plastics research, including Laser-Scanning Confocal 
Microscopy ${ }^{14,16}$ and widefield epifluorescence microscopy ${ }^{17,18}$. Consequently, with conventional light microscopy, nanoplastics are typically only visualized as diffraction limited spots and cannot be precisely localized in tissues nor distinguished from free dye or autofluorescence ${ }^{14}$. While electron microscopy resolution is well beyond the diffraction limit for light microscopy, electron microscopy lacks the labelling flexibility, matrix flexibility, and the ease of sample preparation of fluorescence microscopy.

Nanoplastic exposure experiments disproportionately employ spherical polystyrene nanoparticles, in part due to the limited commercial availability of other types of labeled nanoplastics ${ }^{19,20}$. These polystyrene spheres are not representative of the diversity of environmentally relevant plastic contamination ${ }^{19,20}$. Techniques have been developed to label microplastics of arbitrary shapes and compositions ${ }^{11}$ and Stimulated Emission Depletion (STED) microscopy has been used to image nano-sized polystyrene latex with pre-loaded proprietary fluorophores ${ }^{21}$. However, methods to fluorescently label nanoplastics of different shapes and polymer types are limited. Consequently, a method to label nanoplastic particles of various shapes and composition would allow more environmentally relevant study of nanoplastics transport, uptake and translocation.

In this work, we developed techniques to label nanoplastics of various shapes, sizes, and polymer types with STED-compatible dyes to image at diffraction-unlimited resolution. We show that

nanoplastics can be labeled either by (1) passive sorption; (2) swell incorporation; or (3) covalent coupling of STED-compatible fluorescence dyes. We display STED-compatible labeling and fluorescent imaging of multiple types of nanoplastics, including secondary nanoplastics, of different shapes and polymer types as well as nano-scale resolution imaging of nanoplastics in Caenorhabditis elegans, a model nematode worm. We expect that these techniques will enable localization and identification of nanoplastic translocation at the nano-scale in cells, tissues, and smaller organisms in exposure experiments.

\section{Nanoplastic labeling and imaging at nanoscale resolution}

a

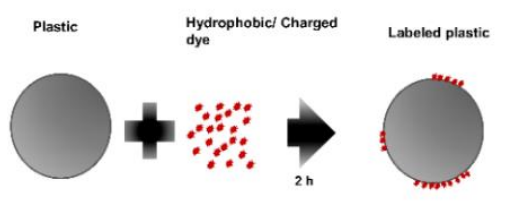

b
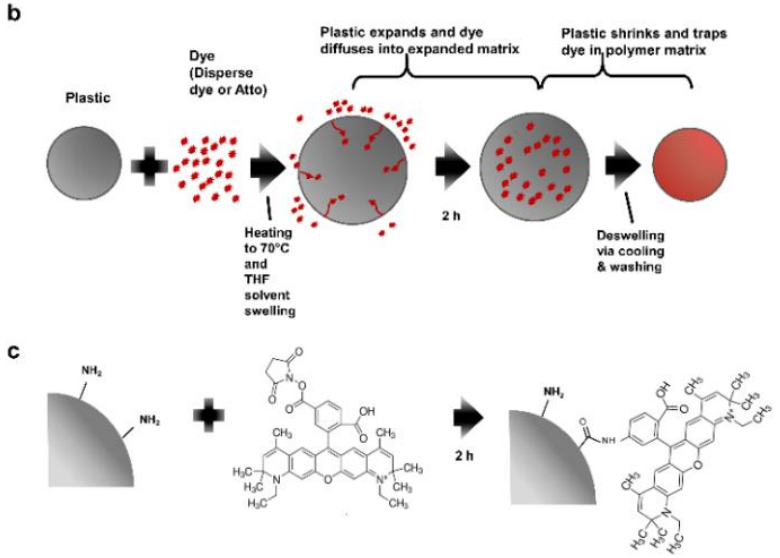
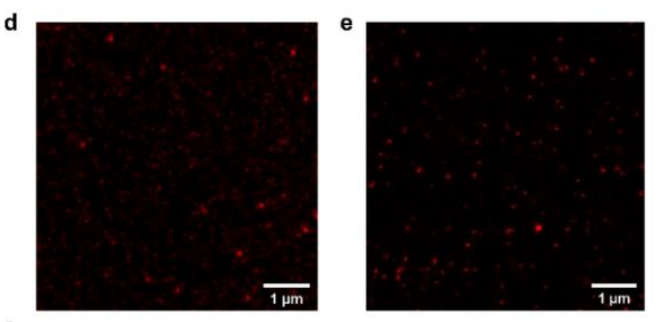

f

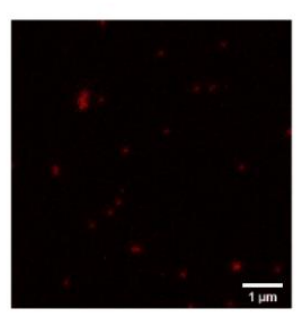

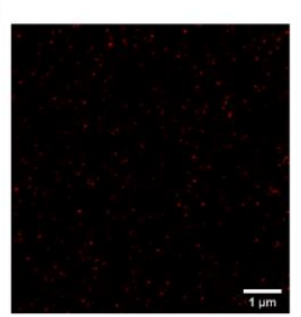

Fig. 1: STED-compatible dye labeling techniques. In this work, all labeled plastics are dialyzed prior to imaging to remove free dye. (a) Passively-labeling with Atto $647 \mathrm{~N}$ where the dye solution and the plastic are simply mixed together; (b) Swell-labeling with Atto 647N/iDye Poly Blue where the plastic is heated in the dye solution to swell the polymer matrix and allow the dye to enter the polymer matrix (with Atto $647 \mathrm{~N}$, THF, a solvent, is added to aid in swelling), after swelling the plastic is cooled and resuspended in DI water to deswell and remove excess dye; (c) Covalent labeling by coupling NHS-functionalized Atto 647N to amine groups on functionalized plastic; (d) STED image of passively-labeled $100 \mathrm{~nm}$ PS beads with Atto 647N; (e) STED image of swell-labeled $100 \mathrm{~nm}$ PS beads 
with Atto 647N; (f) STED image of swell-labeled $100 \mathrm{~nm}$ PS beads with iDye Blue; (g) STED image of covalentlylabeled $100 \mathrm{~nm}$ PS beads with Atto 647N.

Labeling and Resolution

91

STED microscopy achieves sub-diffraction resolution by scanning a sample with two lasers simultaneously: an excitation laser with a circular spot and an overlapping depletion laser with a donut-shaped $\operatorname{spot}^{17}$. The depletion laser suppresses fluorescence emission from the periphery of the excitation laser spot, effectively decreasing the size of the emission spot which improves resolution beyond the diffraction limit. This results in an order of magnitude improvement in resolution. However, the dye compatibility of STED microscopy is limited due to the need for the dye to efficiently undergo stimulated emission and to have high photostability to limit excessive photobleaching ${ }^{22,23}$. Nevertheless, multiple colours of fluorescent dyes compatible with STED microscopy are available ${ }^{24}$. Still, the suitability of these dyes to labeling nanoplastics of different shapes and polymer types has not yet been established.

We tested labeling with Atto $647 \mathrm{~N}$ either via passive sorption, heat/solvent swelling ${ }^{11}$ or covalent coupling (Fig. 1a-c). We also tested swell labeling with iDye Poly Blue ("iDye"). The methods to label nanoplastic that we describe here all successfully labeled nanoplastic for STED imaging (Fig. 1d-g). Fig. 2 compares identical fields of view imaged with standard laser-scanning confocal imaging with those obtained using STED imaging. As expected, the resolution of the laser-scanning confocal images was diffraction-limited. Individual particles appear as a diffraction-limited spot significantly larger than their actual size. When particles are close to each other, multiple particles appear as a single body rather than distinct particles. In contrast, with STED imaging using Atto $647 \mathrm{~N}$, the size and shape of individual nanoplastic particles as small as $50 \mathrm{~nm}$ can be resolved as shown by the images (Fig. 2) and corresponding point-spread functions (Supplementary Fig. 1). Consequently, STED imaging can detect single nanoplastic particles provided that they are in the field of view of the microscope. 


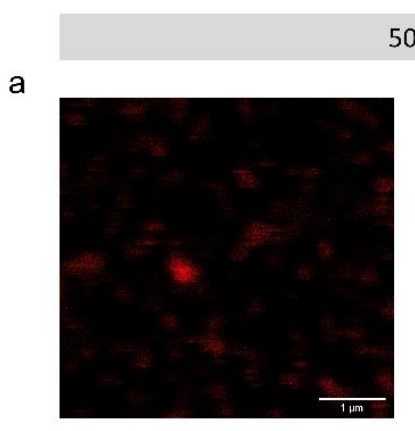

e

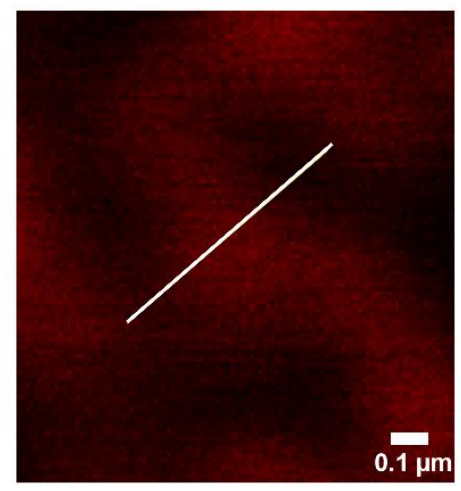

h

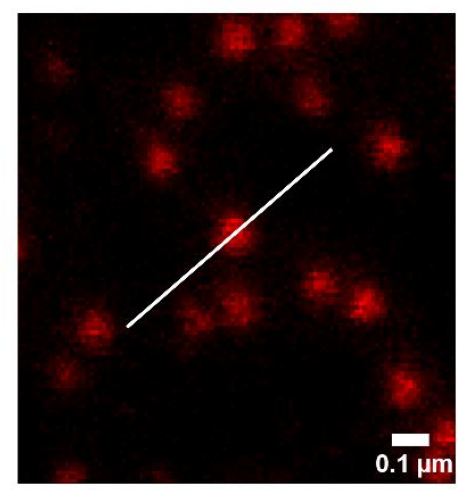

$50 \mathrm{~nm}$

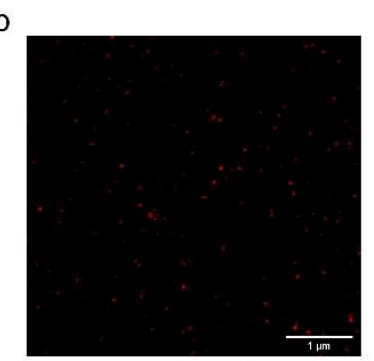

$100 \mathrm{~nm}$

$c$

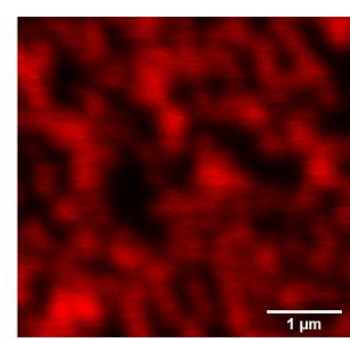

d

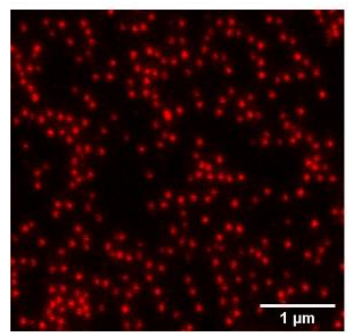

f

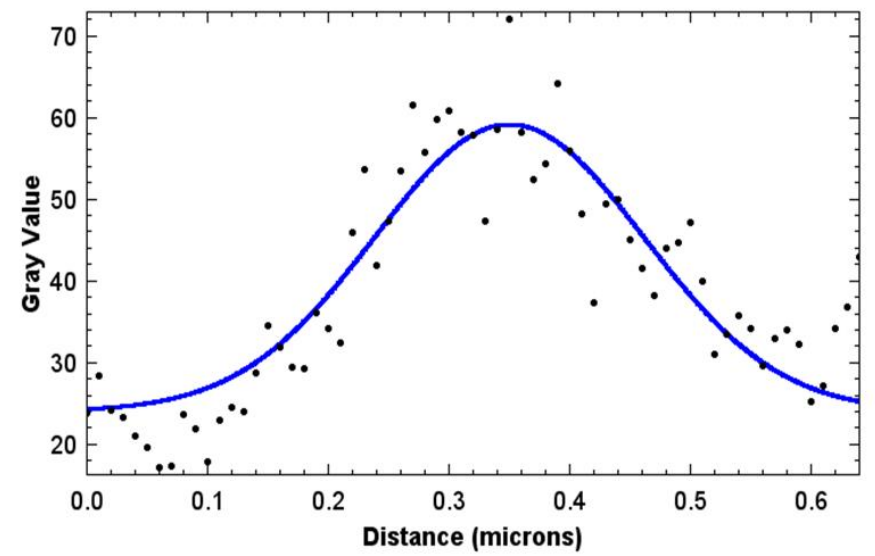

i

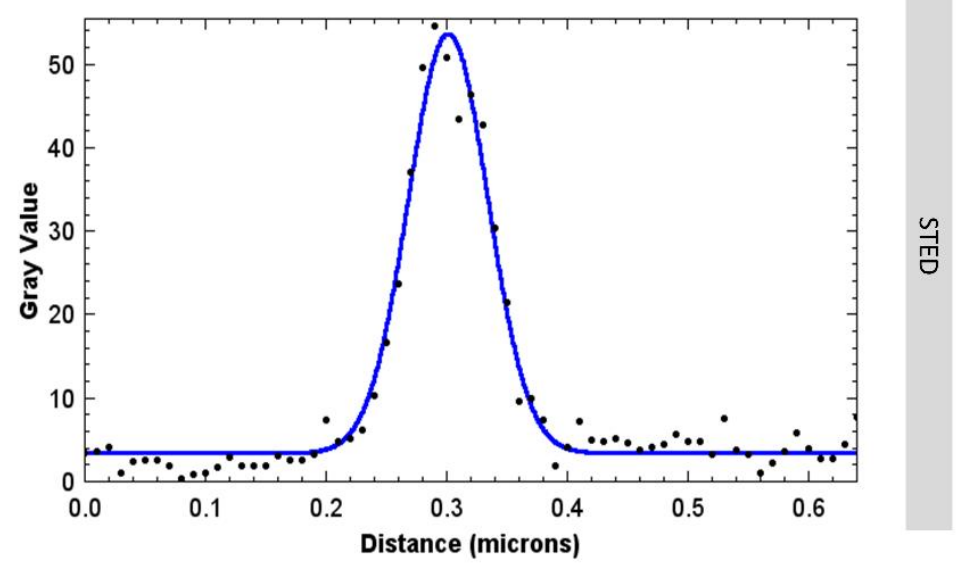

Fig. 2: Comparison of Laser-Scanning Confocal images and STED images of identical fields of view where the nanoplastics are passively labeled with Atto 647N. (a) Confocal image of $50 \mathrm{~nm}$ polystyrene beads; (b) STED image of $50 \mathrm{~nm}$ polystyrene beads; (c) Confocal image of $100 \mathrm{~nm}$ polystyrene beads; (d) STED image of $100 \mathrm{~nm}$ polystyrene beads; (e) Close up confocal image of $100 \mathrm{~nm}$ polystyrene beads; (f) Point spread function and Gaussian fit of $100 \mathrm{~nm}$ polystyrene bead with confocal imaging; (h) Close up STED image of $100 \mathrm{~nm}$ polystyrene beads; (i) Point spread function and Gaussian fit of $100 \mathrm{~nm}$ polystyrene bead with STED imaging.

STED imaging with iDye also showed a resolution improvement over conventional laserscanning confocal microscopy. The point spread functions of STED images are shown in Supplementary Fig. 1-4. However, the resolution with iDye labeling (Supplementary Fig. 3) was lower compared to Atto 647N. This result is unsurprising since Atto $647 \mathrm{~N}$ is well known to perform well with STED microscopy ${ }^{23}$ whereas iDye is a repurposed fabric dye, not a purposedesigned fluorophore. Particles dyed with iDye were also not as bright as those dyed with Atto 
$647 \mathrm{~N}$ and thus required 5 times more intense excitation light and twice the pixel dwell time to achieve comparable signal strengths (Supplementary Table 1).

\section{Longevity}

We tested the longevity of the labeled nanoplastic particles in both water and oil to simulate the polar and non-polar environments that the particles might experience pre- and postinternalization by organisms. The ability to visualize particles at diffraction-unlimited resolution was maintained in the particles labeled using the techniques we present here for typical exposure timescales. Particularly, all the methods that employ Atto $647 \mathrm{~N}$ remain visible for at least 49 days (Fig. 3a-f). While there was variability day to day in the precise average signal intensity, at all points during this test, the nanoplastic particles were clearly visible with STED imaging throughout the duration of the test. Surprisingly, even plastics labeled passively with Atto $647 \mathrm{~N}$ were stable in oil (Fig. 3b), a non-polar environment. Suspending the plastics in mineral oil did not significantly diminish the fluorescent signal localized to the plastic particle. Moreover, we did not detect a fluorescent signal in the oil phase surrounding plastic particles (directly measured with fluorescence microscopy), indicating that dye transfer to the oil phase is limited.
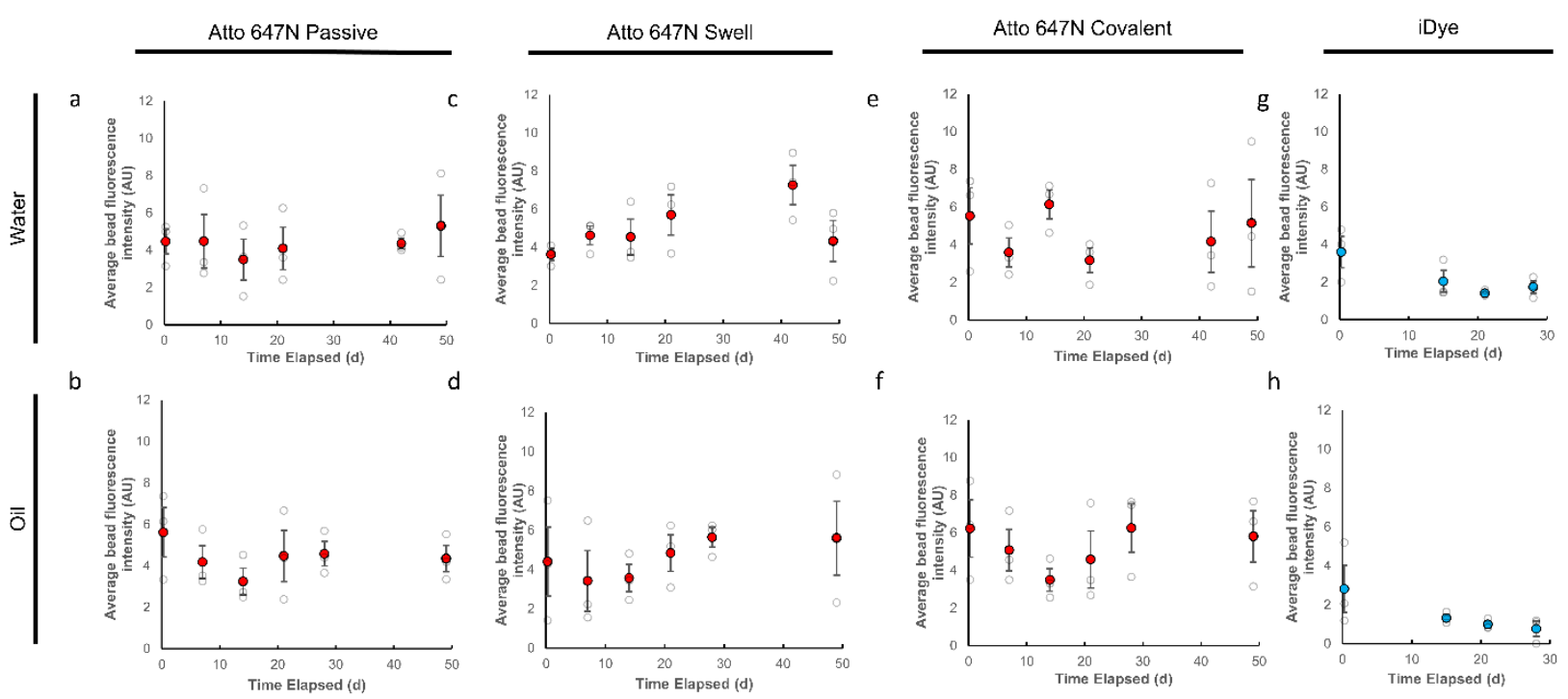

Fig. 3: Longevity of different STED-compatible labeling techniques using $100 \mathrm{~nm}$ beads. Passive labeling with Atto $647 \mathrm{~N}$ in (a) DI water and (b) mineral oil; Swell labeling with Atto 647N in (c) DI water and (d) mineral oil; Covalent labeling with NHS-functionalized Atto $647 \mathrm{~N}$ to amine groups on the particle surface in (e) DI water and (f) mineral oil; Swell labeling with iDye Poly Blue in (g) DI water and (h) mineral oil. Each point represents the average single particle grey value for STED images of triplicate samples acquired with identical settings within treatments. Error bars represent standard error.

The longevity of passively labeled nanoplastics with Atto 647N contrasts with that of Nile Red, a dye more commonly used to stain plastics ${ }^{12,25}$. Previous work ${ }^{11}$ shows that plastics passively stained with Nile Red, do not retain the dye when exposed to mineral oil. This suggests that Atto $647 \mathrm{~N}$ has a higher affinity to plastic surfaces compared to Nile Red and that the mechanism by which Atto $647 \mathrm{~N}$ sorbs to plastics is not solely via hydrophobic interaction. Other potential mechanisms of sorption could include interaction between the plastic surface and the charged regions of Atto $647 \mathrm{~N}$ and/or passive diffusion and subsequent intercalation into the polymer matrix. 
To further test the longevity of the labeling in different biologically and environmentally-relevant media and conditions, we also tested the longevity of the different Atto $647 \mathrm{~N}$ labeling methods on $50 \mathrm{~nm}$ polystyrene beads in pitcher plant digestive fluid, soil water, $2.5 \mathrm{pH}$ hydrochloric acid, and at elevated temperature $\left(40^{\circ} \mathrm{C}\right)$ over 21 days (Fig. 4). Similar to the tests in water and oil, we found that we were able to visualize the labeled plastics over the period of exposure.
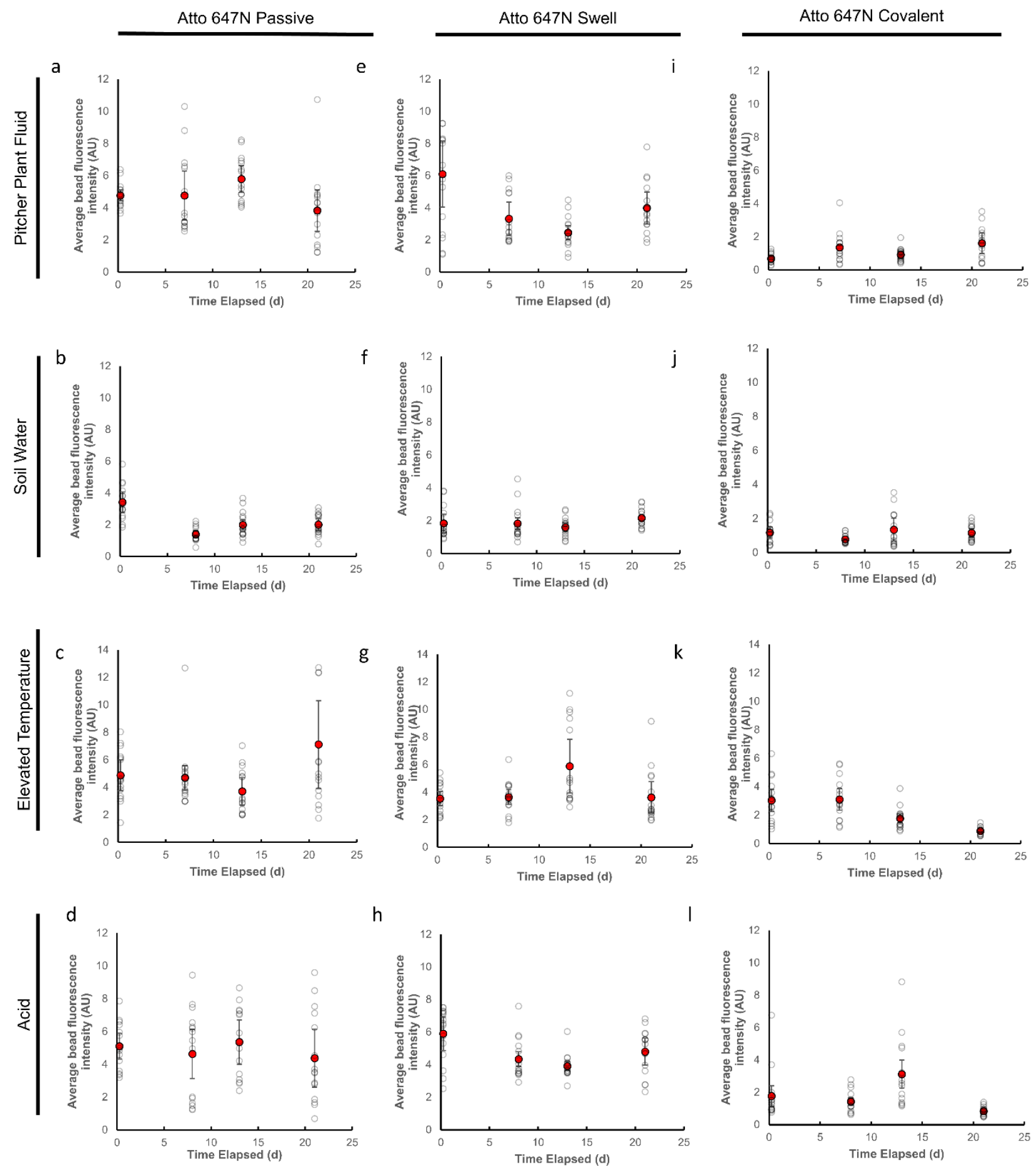

Fig. 4: Longevity of different STED-compatible labeling techniques using $50 \mathrm{~nm}$ beads. Passive labeling with Atto $647 \mathrm{~N}$ in (a) pitcher plant fluid; (b) soil water; (c) elevated temperature $\left(40^{\circ} \mathrm{C}\right)$; and (d) hydrochloric acid ( $\left.\mathrm{pH} 2.5\right)$. Swell labeling with Atto $647 \mathrm{~N}$ in (e) pitcher plant fluid; (f) soil water; (g) elevated temperature $\left(40{ }^{\circ} \mathrm{C}\right)$; and (h) 
hydrochloric acid ( $\mathrm{pH}$ 2.5). Covalent labeling with Atto 647N in (i) pitcher plant fluid; (j) soil water; (k) elevated temperature $\left(40^{\circ} \mathrm{C}\right)$; and (1) hydrochloric acid ( $\left.\mathrm{pH} 2.5\right)$. Each point represents the average single particle grey value for STED images of triplicate samples acquired with identical settings within treatments. Error bars represent standard error.

Theoretically, swell and covalent labeling potentially provide greater labeling longevity compared to passive labeling alone. However, these techniques are comparatively more complicated to carry out. Particularly, with covalent labeling, specific functional groups on the surface of the plastic are required for labeling. Nevertheless, depending on the target application, swell or covalent labeling may be useful when greater resistance to external solvents is desirable.

As with any fluorescent labeling method, the fluorescent signal will be susceptible to photobleaching ${ }^{11}$. Consequently, if experimental exposures require intense light, duration of the exposure while maintaining a fluorescent signal may be more limited. Nevertheless, Atto $647 \mathrm{~N}$ is generally a photostable dye ${ }^{26}$. Furthermore, despite the resolution advantages that we show, STED microscopy results in a greater degree of photobleaching compared to conventional widefield or confocal fluorescence microscopy since bleaching can also occur via stimulated emission in addition to fluorescence ${ }^{23}$. Consequently, the ability to observe dynamics and image multiple z-slices is limited, in a sample dependent manner, compared to conventional laserscanning confocal or widefield microscopy. Moreover, to achieve nanoscale resolution, high numerical aperture objectives must be used. Typically, these objectives have limited working distances. Consequently, the maximum imaging depth with STED microscopy while maintaining high resolution is limited and is most suited for imaging cells, tissue sections, small anatomy, or micro-scale organisms.

In terms of performance with STED microscopy, Atto 647N was superior to iDye in brightness, longevity and resolution. Nevertheless, dyeing with iDye may be more practical when cost is a concern. In contrast to Atto $647 \mathrm{~N}$, which currently costs $\$ 288$ CAD per mg, iDye Poly Blue can be purchased for less than $\$ 0.50$ CAD per g. Nonetheless, typically, a much lower amount of Atto $647 \mathrm{~N}$ would be required to effectively label nanoplastics compared to iDye Poly Blue.

\section{Labeling and imaging various types of nanoplastic}

While techniques have been developed to label microplastics of arbitrary shapes and compositions ${ }^{11}$, methods to fluorescently label nanoplastics of different shapes and polymer types are limited. Addressing this limitation, our methods are compatible with different shapes and compositions of nanoplastic, beyond commercially available spherical nanoplastics (Fig. 5). With STED microscopy, we were able to resolve the shapes of secondary nanoplastics produced by heating expanded polystyrene to $90{ }^{\circ} \mathrm{C}$ for 7 days (Fig. 5b) as well as polystyrene sanding debris (Fig. 5d). As confirmed by SEM (Supplementary Fig. 9), the secondary nanoplastics produced by heating are roughly spherical while those produced by sanding are irregular in shape. The images in Fig. 5a,c show that such particle visualization was not possible with conventional confocal fluorescence microscopy. We also tested imaging with commercially-available PTFE nanoparticles (Fig. 5e,f) as a low surface energy plastic and PMMA nanoparticles (Fig. 5g,h) as a relatively high surface energy plastic ${ }^{27}$. The PTFE particles are representative of engineered PTFE nanoparticles used for lubrication and hydrophobic coatings. PMMA (also known as "Plexiglas") is widely used as a glass substitute for transparent windows. 

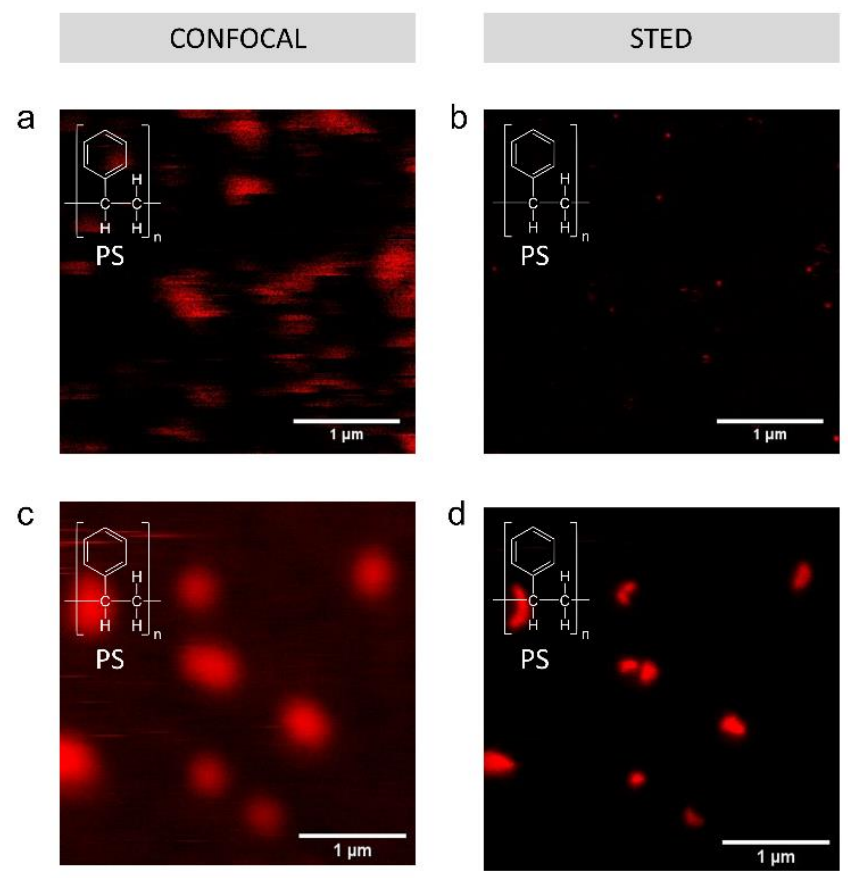

e
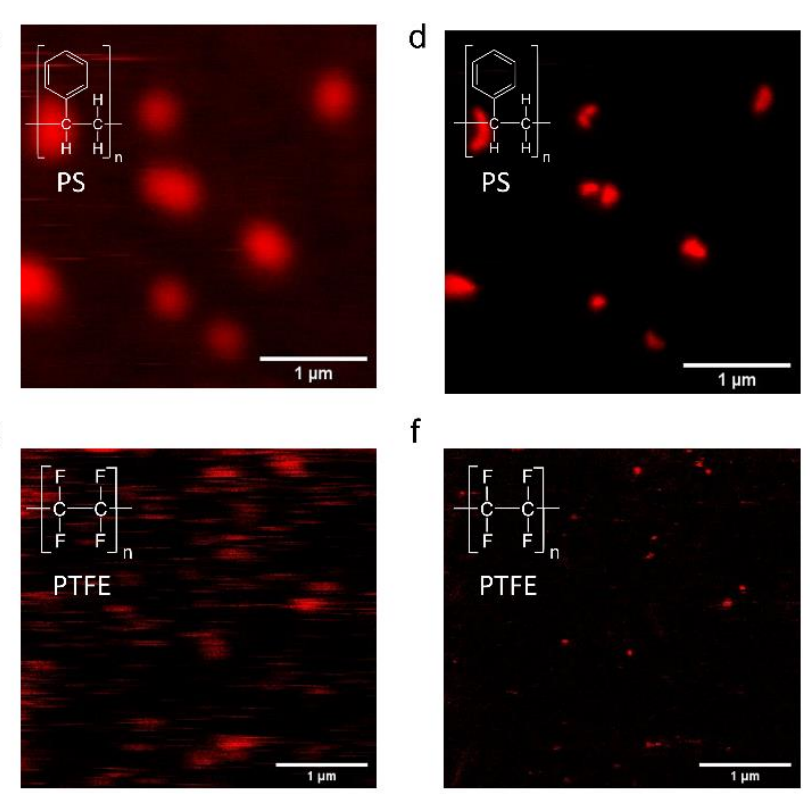

f

$\mathrm{g}$
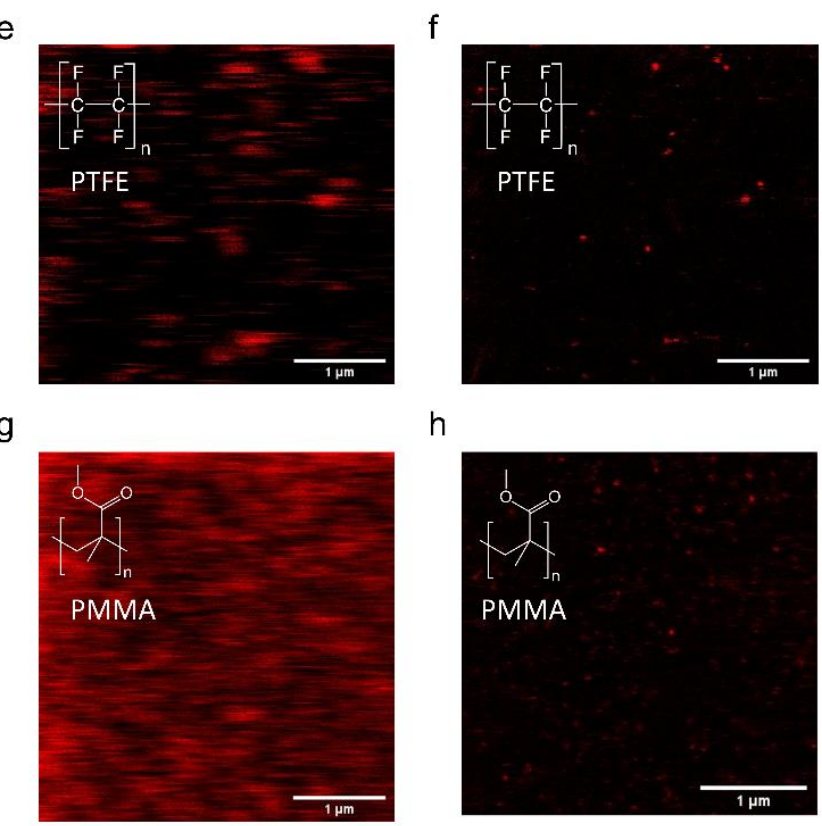

$\mathrm{h}$

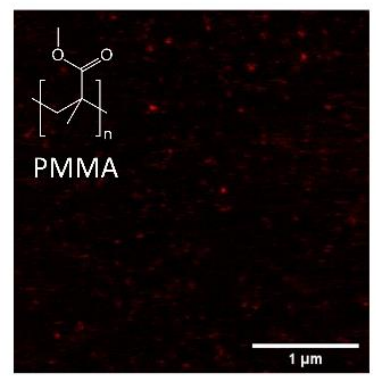

Fig. 5: Passive labeling and fluorescent imaging of various nanoplastic types with Atto 647N. Confocal (a) and STED (b) image of debris released from an expanded polystyrene plate exposed to $90{ }^{\circ} \mathrm{C}$ in DI water; (c) Confocal and (d) STED image of sanding debris from a polystyrene Petri dish; (e) Confocal and (f) STED image of PTFE particles; (g) Confocal and (h) STED image of PMMA particles. Confocal and STED images are of the same fields of view.

\section{Application to C. elegans exposure}

To test the ability of this technique to image nanoplastics in a model organism, as a proof of concept, we exposed C. elegans $\mathrm{KWN} 117^{28}$ to $100 \mathrm{ppm}$ of $50 \mathrm{~nm}$ polystyrene beads passively labeled with Atto 647N (Fig. 6). We used the $50 \mathrm{~nm}$ polystyrene beads due to their availability in sufficient quantities to conduct the exposures as well as a more challenging size to detect. KWN117 is a transgenic strain that expresses Green Fluorescent Protein (GFP) in the body wall and mCherry in apical intestinal membrane cells ${ }^{28}$. As when imaging the nanoplastics alone, 
STED imaging allows visualization of nanoplastic along sections of the digestive tract including the mouth (Fig. 6a,b,c), pharynx (Fig. 6d,e,f), and intestine (Fig. 6g,h,i). While 100 ppm is

a

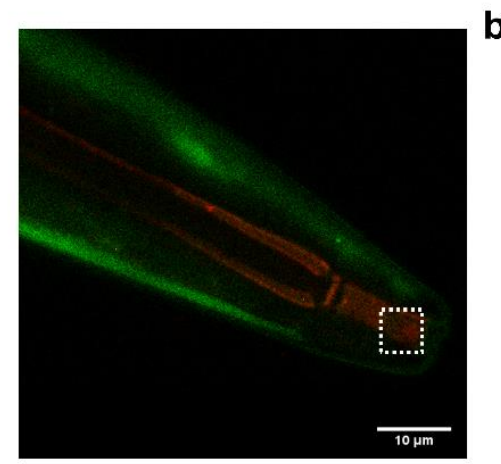

d

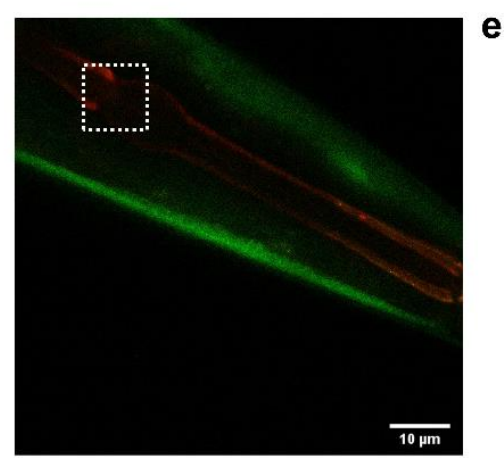

g

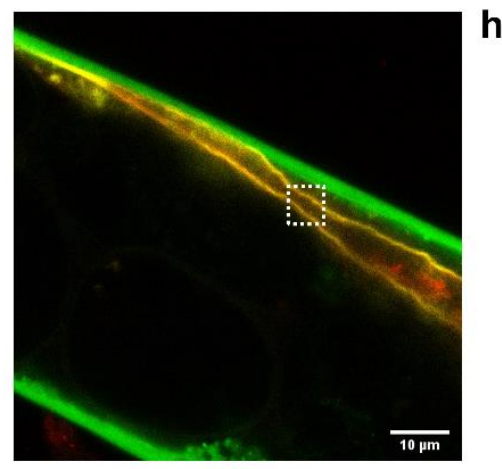

b
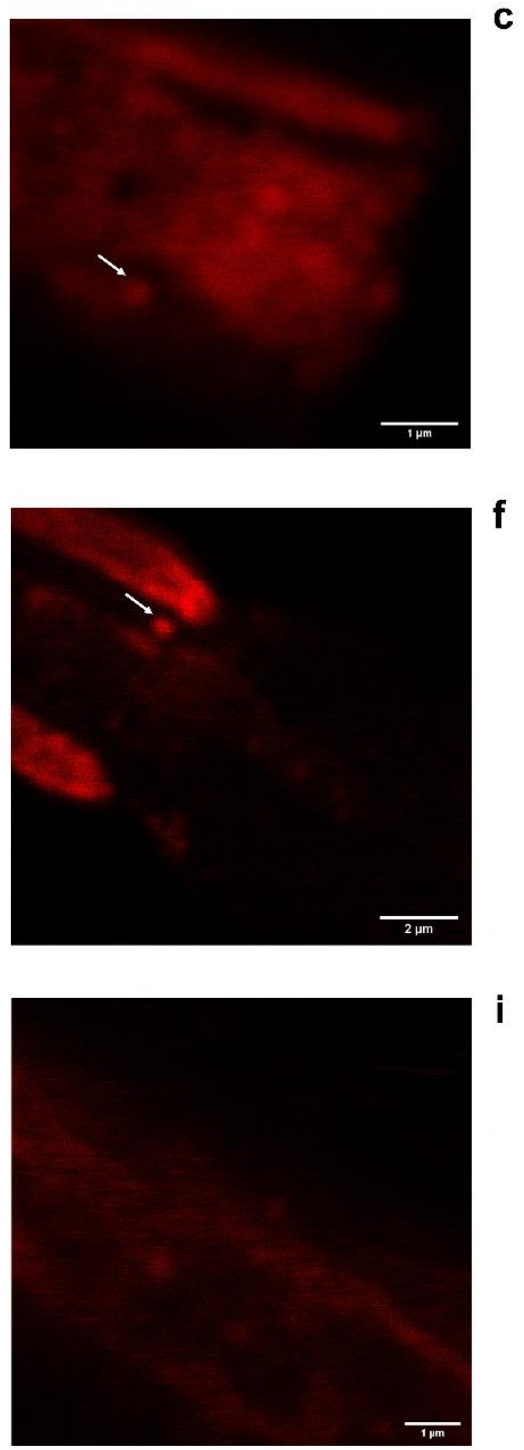
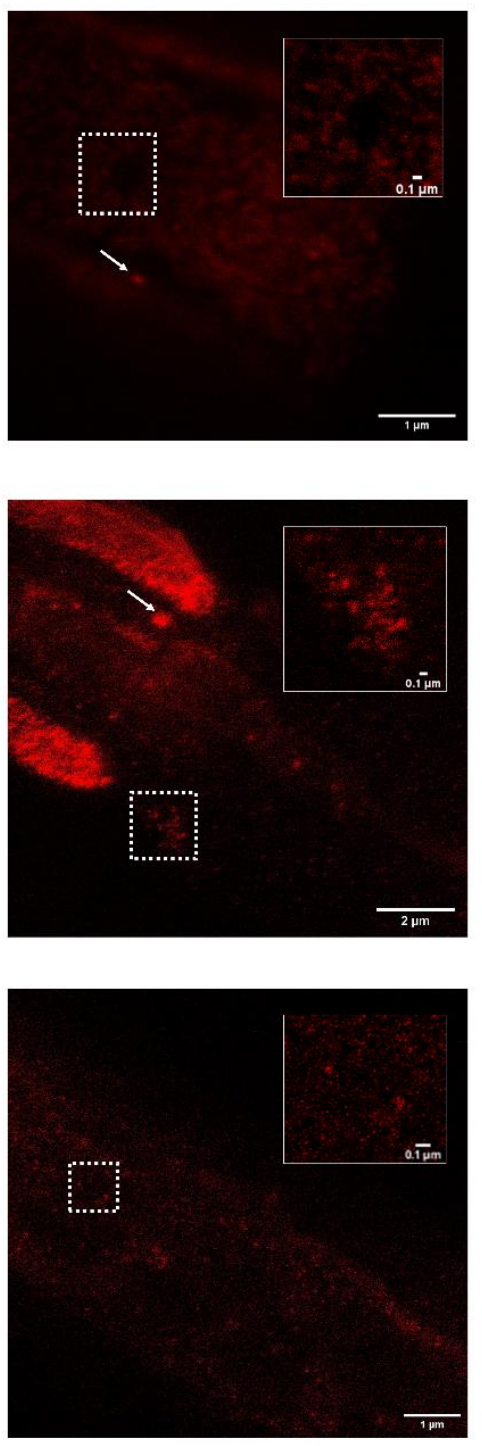

Fig. 6: Imaging $50 \mathrm{~nm}$ polystyrene nanoplastics passively labeled with Atto $647 \mathrm{~N}$ (red) in C. elegans KWN117 adult expressing GFP (green) in the body wall and mCherry (yellow) in the apical intestinal membrane. Confocal overview, and high resolution of confocal and STED images of scanned area indicated by white boxes for parts of the digestive track in the mouth (a,b,c), pharynx (d,e,f), and intestine (g,h,i). Insets in STED images correspond to the areas indicated by white boxes. Arrows indicate potential false positive signal from confocal imaging where STED imaging reveals that the signal is inconsistent with $50 \mathrm{~nm}$ particles. 
We expect that for typical organism exposure experiments, including the imaging in C. elegans we show here, STED microscopy combined with passive staining with Atto $647 \mathrm{~N}$ would generally be a convenient method of observing nanoplastics fluorescently without significantly compromising labeling longevity. Overall, the ability to precisely localize the distribution of nanomaterials, including nanoplastics, in organisms may help facilitate study into the lethal and sub-lethal effects observed in exposure experiments. Localization can also provide insight into longer term effects of exposure that may not be detected over relatively short experimental timescales. Specifically, translocation of materials to certain parts of organisms can be indicative of long-term effects. Since our methods are compatible with different shapes and compositions of nanoplastic, these techniques will allow researchers to more thoroughly explore the impact of shape, size, and composition of nanoplastics on toxicity and translocation in live organisms. This broad compatibility of STED microscopy to localize fluorescently-labeled nanomaterials at nanoscale resolution can be further extended to better understand the environmental toxicology of nanomaterials beyond nanoplastics. While STED microscopy has been applied to understand the interaction of engineered nanomaterials with cells in vitro in the biomedical context ${ }^{31-33}$, our work demonstrates the utility of STED microscopy to study nanomaterial interactions with organisms in environmentally-relevant exposure scenarios and with environmentally-relevant contaminants.

\section{Methods}

\section{Nanoplastic sources}

Plain polystyrene spheres with a nominal diameter of $100 \mathrm{~nm}$ (actual diameter averaged $88 \mathrm{~nm}$ according to manufacturer specifications) and $50 \mathrm{~nm}$ (actual diameter averaged $50 \mathrm{~nm}$ according to manufacturer Dynamic Light Scattering specifications) were purchased from Polysciences Inc. Amine-functionalized $100 \mathrm{~nm}$ (actual diameter averaged $113 \mathrm{~nm}$ according to manufacturer Dynamic Light Scattering specifications) polystyrene spheres was purchased from Polysciences Inc. Plain $50 \mathrm{~nm}$ (actual diameter averaged $50 \mathrm{~nm}$ according to manufacturer specifications) Poly(methyl methacrylate) spheres were purchased from Phosphorex Inc. These commercially available spherical particles are typical of those used in most current nanoplastic studies ${ }^{14,16}$. A dispersion of polytetrafluoroethylene (PTFE) nanoparticles (Teflon ${ }^{\mathrm{TM}}$ 30B) was purchased from Polysciences Inc. This dispersion has typical average particle diameters around $200 \mathrm{~nm}$ but are not monodisperse and contain a variety of particle sizes including much smaller particles.

We also obtained nano-sized debris from plastic labware and consumer items using a variety of weathering methods. Debris was obtained from a polystyrene Petri dish by mechanical abrasion with a $100 \mathrm{~nm}$ diamond lapping film (3M) and from an expanded polystyrene plate by submerging it in approximately $1 \mathrm{~L}$ of DI water at approximately $90{ }^{\circ} \mathrm{C}$ for 7 days and sampling the water by pipetting into a glass vial. These secondary nanoplastic particles represent nanoplastics that can be released from common single-use plastic consumer goods $\$, 34,35$.

\section{Labeling nanoplastics}

Passive Staining of Nanoplastics. We labeled nanoplastic via passive sorption with Atto 647N (Sigma 04507) (ex. $646 \mathrm{~nm} / \mathrm{em} .664 \mathrm{~nm}$ ). We chose to use Atto 647N because of its good STED performance with a $775 \mathrm{~nm}$ depletion laser. To passively label nanoplastic, we suspended $0.125 \%$ w/v nanoplastic in a solution of $2 \mathrm{mg} / \mathrm{L}$ Atto $647 \mathrm{~N}$ in DI water for $2 \mathrm{~h}$ at room temperature $(\sim 25$ $\left.{ }^{\circ} \mathrm{C}\right)$. The excess dye was removed from the suspension via dialysis using 12-14 kDa molecular weight cutoff (MWCO) dialysis membranes (Frey Scientific) for 7 days with daily water changes in stirred $2 \mathrm{~L}$ glass beakers. 
Swell Incorporation of Dye to Nanoplastics. We also adapted the microplastic dyeing methods developed previously by Karakolis et al. ${ }^{11}$ to label nanoplastics with iDye (Jacquard Products), an inexpensive commercially available fabric dye. While we expected that iDye would be inferior to Atto $647 \mathrm{~N}$ in terms of fluorescence imaging, we tested it due to the convenience and low cost of labeling with the iDye Poly Blue ${ }^{11}$. Briefly, nanoplastics were incubated in a $0.1 \mathrm{mg} / \mathrm{mL}$ solution of iDye Poly Blue in DI water heated to $70{ }^{\circ} \mathrm{C}$ for $2 \mathrm{~h}$ followed by cooling to room temperature. During the heating, the dye is able to diffuse into the polymer matrix due to thermal swelling. Since iDye Poly is only sparingly soluble, the excess dye was removed via centrifugation $(12,000 \mathrm{~g}$ for $45 \mathrm{~min})$, washing the dyed particles twice with $25 \%$ isopropanol and three times with DI water. We also tested a similar method using Atto $647 \mathrm{~N}$ in place of iDye Poly Blue. However, for swell incorporation of Atto $647 \mathrm{~N}$, we added $10 \%$ tetrahydrofuran to the dye solution to aid in the swelling in addition to heating to $70{ }^{\circ} \mathrm{C}$. We added the tetrahydrofuran since Atto $647 \mathrm{~N}$ is not packaged with dispersants or other agents which are typically included in commercial fabric dye formulations. We were also able to remove excess dye using dialysis since Atto $647 \mathrm{~N}$ is more water soluble ${ }^{36}$.

Covalent Coupling of Dye to Nanoplastics. We coupled N-hydroxysuccinimide (NHS)terminated Atto $647 \mathrm{~N}$ (Sigma 18373) to amine-functionalized plastic by incubating $0.125 \% \mathrm{w} / \mathrm{v}$ nanoplastic in a solution of $2 \mathrm{mg} / \mathrm{L}$ Atto $647 \mathrm{~N}$ in phosphate buffered saline at $\mathrm{pH} 7.4$. At this $\mathrm{pH}$, the NHS group reacts with the amine groups on the surface of the plastic, covalently binding the dye to the plastic ${ }^{37}$. The excess dye was removed from the suspension via dialysis using a 12-14 $\mathrm{kDa}$ MWCO dialysis membranes (Frey Scientific) for 7 days with daily water changes in stirred 2 L glass beakers ${ }^{13}$.

\section{Fluorescence Imaging}

We completed all imaging using an Abberior Expert Line STED microscope using an Olympus Plan-Apo 100x/1.40NA Oil immersion objective. We used a $640 \mathrm{~nm}$ laser for excitation and a 775 $\mathrm{nm}$ laser for depletion. The microscope was equipped with avalanche photodetectors. We performed both standard laser-scanning confocal and STED imaging on the same microscope. All image analysis was done in ImageJ.

\section{Oil Longevity Testing}

To test the longevity of the dye-labeled nanoplastic in a non-polar solvent, we mounted labeled $100 \mathrm{~nm}$ (nominal) polystyrene beads in mineral oil (Sigma M5904) on sealed glass microscope slides stored at room temperature $\left(25^{\circ} \mathrm{C}\right)$ in the dark. Specifically, we spread a $5 \mu \mathrm{L}$ drop of the nanoplastic suspension on glass slides with a pipette tip, allowed the water to dry and then applied a $20 \mu \mathrm{L}$ drop of mineral oil onto the slide. We then covered the oil with a \#1.5 coverslip and sealed the edges with CoverGrip Coverslip Sealant (Biotium, Inc.). Samples were imaged at each time point using identical imaging settings (10\% excitation laser power, $50 \%$ depletion laser power, $10 \mathrm{~nm}$ pixel size, $10 \mu$ s dwell time and 1.0 AU pinhole-See Supplementary Table 1 for imaging settings).

\section{Aqueous Media Longevity Testing}

As water is more volatile than the mineral oil we used, we tested longevity in aqueous solutions, including simulated environmental media, in $1 \mathrm{~mL}$ suspensions of labeled nanoplastic in glass vials at room temperature $\left(\sim 25^{\circ} \mathrm{C}\right)$ or at elevated temperature $\left(40^{\circ} \mathrm{C}\right)$. Pitcher plant fluid was a 
gift from Brad's Greenhouse (Vancouver Island, British Columbia, Canada). Soil water was obtained by mixing $200 \mathrm{~mL}$ of DI water with $100 \mathrm{~g}$ of dry agricultural soil from McGill University's MacDonald Campus (Sainte-Anne-de-Bellevue, Quebec, Canada) for $48 \mathrm{~h}$ and taking the supernatant after the soil was allowed to settle.

At each time point, we mounted a sample from each suspension onto microscope slides for imaging. Specifically, we spread a $5 \mu \mathrm{L}$ drop of the nanoplastic suspension on glass slides with a pipette tip, allowed the water to dry and then applied a $20 \mu \mathrm{L}$ drop of water onto the slide. Samples were imaged at each time point using identical imaging settings $(10 \%$ excitation laser power, $50 \%$ depletion laser power, $10 \mathrm{~nm}$ pixel size, $10 \mu$ s dwell time and 1.0 AU pinhole for $100 \mathrm{~nm}$ particles and $20 \%$ excitation laser power, $50 \%$ depletion laser power, $5 \mathrm{~nm}$ pixel size, $10 \mu$ s dwell time and 1.0 AU pinhole for $50 \mathrm{~nm}$ particles and -See Supplementary Table 1 for imaging settings).

\section{C. elegans culture and exposure}

C. elegans strain KWN117 worms were obtained from the Caenorhabditis Genetics Center at the University of Minnesota and maintained at $23{ }^{\circ} \mathrm{C}$ on Nematode Growth Medium (NGM) plates seeded with $E$. coli OP50 as a food source. KWN117 expresses GFP (488 nm excitation/507 nm emission) in the body wall as well as mCherry (587 nm excitation/610 nm emission) in the apical intestinal membrane ${ }^{28}$.

Prior to exposing the worms to nanoplastic, worms were washed from NGM agar plates with 5 $\mathrm{mL}$ of M9 buffer and washed with M9 buffer three times by allowing the worms to settle in 15 $\mathrm{mL}$ centrifuge tubes for $\sim 10 \mathrm{~min}$ and replacing the supernatant with fresh M9 buffer. To expose the worms to nanoplastic, the worms were resuspended in $100 \mu \mathrm{L}$ of M9 buffer containing nanoplastics and then pipetted onto NGM agar plates. Control worms were prepared similarly but were resuspended in M9 buffer with dialyzed dye solution before pipetting on NGM agar plates. We expect that the dye would be largely removed from the solution with this process. For imaging, the worms were washed off the NGM with M9 buffer and immobilized in low-melting point agarose on microscope slides covered with \#1.5 coverslips.

\section{References}

1. Geyer, R., Jambeck, J. R. \& Law, K. L. Production, use, and fate of all plastics ever made. 25-29 (2017).

2. Moore, C. J. Synthetic polymers in the marine environment: A rapidly increasing, longterm threat. Environ. Res. 108, 131-139 (2008).

3. Thompson, R. C., Moore, C. J., Saal, F. S. V. \& Swan, S. H. Plastics, the environment and human health: Current consensus and future trends. Philos. Trans. R. Soc. B Biol. Sci. 364, 2153-2166 (2009).

4. Thompson, R. C., Swan, S. H., Moore, C. J. \& Vom Saal, F. S. Our plastic age. Philos. Trans. R. Soc. B Biol. Sci. 364, 1973-1976 (2009).

5. Magrì, D. et al. Laser ablation as a versatile tool to mimic polyethylene terephthalate nanoplastic pollutants: Characterization and toxicology assessment. ACS Nano 12, 76907700 (2018).

6. Dawson, A. L. et al. Turning microplastics into nanoplastics through digestive fragmentation by Antarctic krill. Nat. Commun. 9, 1001 (2018).

7. Hernandez, L. M. et al. Plastic Teabags Release Billions of Microparticles and Nanoparticles into Tea. Environ. Sci. Technol. 53, 12300-12310 (2019). 
8. Mintenig, S. M., Bäuerlein, P. S., Koelmans, A. A., Dekker, S. C. \& van Wezel, A. P. Closing the gap between small and smaller: towards a framework to analyse nano- and microplastics in aqueous environmental samples. Environ. Sci. Nano (2018). doi:10.1039/C8EN00186C

9. Silva, A. B. et al. Microplastics in the environment: Challenges in analytical chemistry - A review. Anal. Chim. Acta 1017, (2018).

10. Nguyen, B. et al. Separation and Analysis of Microplastics and Nanoplastics in Complex Environmental Samples. Acc. Chem. Res. 52, 858-866 (2019).

11. Karakolis, E. G., Nguyen, B., You, J. B., Rochman, C. M. \& Sinton, D. Fluorescent Dyes for Visualizing Microplastic Particles and Fibers in Laboratory-Based Studies. Environ. Sci. Technol. Lett. 6, 334-340 (2019).

12. Cole, M. A novel method for preparing microplastic fibers. Sci. Rep. 6, 34519 (2016).

13. Pikuda, O., Xu, E. G., Berk, D. \& Tufenkji, N. Toxicity Assessments of Micro- and Nanoplastics Can Be Confounded by Preservatives in Commercial Formulations. Environ. Sci. Technol. Lett. 6, 21-25 (2019).

14. Schür, C. et al. When Fluorescence Is not a Particle: The Tissue Translocation of Microplastics in Daphnia magna Seems an Artifact. Environ. Toxicol. Chem. 38, 14951503 (2019).

15. Catarino, A. I., Frutos, A. \& Henry, T. B. Use of fluorescent-labelled nanoplastics (NPs) to demonstrate NP absorption is inconclusive without adequate controls. Sci. Total Environ. 670, 915-920 (2019).

16. Sun, X. D. et al. Differentially charged nanoplastics demonstrate distinct accumulation in Arabidopsis thaliana. Nat. Nanotechnol. (2020). doi:10.1038/s41565-020-0707-4

17. Hell, S. W. \& Wichmann, J. Breaking the diffraction resolution limit by stimulated emission: stimulated-emission-depletion fluorescence microscopy. Opt. Lett. 19, 780 (1994).

18. Betzig, E. et al. Imaging Intracellular Fluorescent Proteins at Nanometer Resolution. $\mathbf{x}$, 1642-1646 (2006).

19. Rochman, C. M. et al. Rethinking microplastics as a diverse contaminant suite. Environ. Toxicol. Chem. 38, 703-711 (2019).

20. Wagner, S. \& Reemtsma, T. Things we know and don't know about nanoplastic in the environment. Nat. Nanotechnol. 14, 300-301 (2019).

21. Harke, B. et al. Resolution scaling in STED microscopy. Opt. Express 16, 4154 (2008).

22. Wurm, C. A. et al. Novel red fluorophores with superior performance in STED microscopy. Opt. Nanoscopy 1, 1-7 (2012).

23. Oracz, J., Westphal, V., Radzewicz, C., Sahl, S. J. \& Hell, S. W. Photobleaching in STED nanoscopy and its dependence on the photon flux applied for reversible silencing of the fluorophore. Sci. Rep. 7, 1-14 (2017).

24. Sednev, M. V., Belov, V. N. \& Hell, S. W. Fluorescent dyes with large Stokes shifts for super-resolution optical microscopy of biological objects: A review. Methods Appl. Fluoresc. 3, (2015).

25. Maes, T., Jessop, R., Wellner, N., Haupt, K. \& Mayes, A. G. A rapid-screening approach to detect and quantify microplastics based on fluorescent tagging with Nile Red. Nat. Publ. Gr. 1-10 (2017). doi:10.1038/srep44501

26. Zheng, Q., Jockusch, S., Zhou, Z. \& Blanchard, S. C. The contribution of reactive oxygen species to the photobleaching of organic fluorophores. Photochem. Photobiol. 90, 448-454 (2014).

27. Park, J. S., Lee, S. M., Joo, B. S. \& Jang, H. The effect of material properties on the stickslip behavior of polymers: A case study with PMMA, PC, PTFE, and PVC. Wear 378-379, 11-16 (2017). 
28. Allman, E., Johnson, D. \& Nehrke, K. Loss of the apical V-ATPase a-subunit VHA-6 prevents acidification of the intestinal lumen during a rhythmic behavior in C. elegans. Am. J. Physiol. - Cell Physiol. 297, 1071-1081 (2009).

29. Scheurer, M. \& Bigalke, M. Microplastics in Swiss Floodplain Soils. Environ. Sci. Technol. 52, 3591-3598 (2018).

30. Fuller, S. \& Gautam, A. A Procedure for Measuring Microplastics using Pressurized Fluid Extraction. Environ. Sci. Technol. 50, 5774-5780 (2016).

31. Schübbe, S. et al. Size-dependent localization and quantitative evaluation of the intracellular migration of silica nanoparticles in Caco-2 cells. Chem. Mater. 24, 914-923 (2012).

32. Tzeng, Y. K. et al. Superresolution imaging of albumin-conjugated fluorescent nanodiamonds in cells by stimulated emission depletion. Angew. Chemie - Int. Ed. 50, 2262-2265 (2011).

33. Pujals, S. \& Albertazzi, L. Super-resolution Microscopy for Nanomedicine Research. ACS Nano 13, 9707-9712 (2019).

34. Lambert, S. \& Wagner, M. Characterisation of nanoplastics during the degradation of polystyrene. Chemosphere 145, 265-268 (2016).

35. Ekvall, M. T. et al. Nanoplastics formed during the mechanical breakdown of daily-use polystyrene products. Nanoscale Adv. 1, 1055-1061 (2019).

36. Hughes, L. D., Rawle, R. J. \& Boxer, S. G. Choose your label wisely: Water-soluble fluorophores often interact with lipid bilayers. PLoS One 9, (2014).

37. Karakolis, E. G. et al. Digestible Fluorescent Coatings for Cumulative Quantification of Microplastic Ingestion. Environ. Sci. Technol. Lett. 5, 62-67 (2018).

\section{Acknowledgments}

General: The authors acknowledge Dr. Rachel Genthial, Kamila Mustafina, Dr. Thomas Stroh, the Integrated Quantitative Biology Initiative, and the Montreal Neurological Institute Microscopy Unit for assistance with STED microscopy. The authors also acknowledge Amin Valiei for assistance with SEM. C. elegans were provided by the CGC, which is funded by NIH Office of Research Infrastructure Programs (P40 OD010440).

Funding: B.N. gratefully acknowledges funding from the Natural Sciences and Engineering Research Council Postdoctoral Fellowships program and the Eugenie Ulmer Lamothe fund in the Department of Chemical Engineering at McGill University. N.T. acknowledges funding from the Canada Research Chairs program, the Natural Sciences and Engineering Research Council of Canada, and the Canada Foundation for Innovation. This Project was supported partially by a financial contribution from Fisheries and Oceans Canada.

Author contributions: B.N. developed the methodology and performed the experiments. B.N. and N.T conceived the concepts and contributed to manuscript drafting and editing.

Competing interests: The authors do not have any competing interests to declare. 
Data Availability: The data that support the findings of this study are available from the corresponding author upon reasonable request. Figure 3 and Supplementary Figures 1-4 have associated raw data. 


\section{Supplementary Information}

516 Supplementary Table 1: STED imaging settings

\begin{tabular}{|c|c|c|c|c|c|}
\hline Sample & $\begin{array}{l}640 \mathrm{~nm} \\
\text { excitation laser } \\
\text { power }\end{array}$ & $\begin{array}{l}775 \mathrm{~nm} \\
\text { depletion } \\
\text { laser power }\end{array}$ & Pixel Size & Dwell Time & Pinhole \\
\hline $\begin{array}{l}50 \mathrm{~nm} \\
\text { polystyrene for } \\
\text { testing resolution }\end{array}$ & $20 \%$ & $100 \%$ & $10 \mathrm{~nm}$ & $10 \mu \mathrm{s}$ & $1.0 \mathrm{AU}$ \\
\hline $\begin{array}{l}100 \mathrm{~nm} \\
\text { polystyrene for } \\
\text { testing resolution }\end{array}$ & $10 \%$ & $50 \%$ & $10 \mathrm{~nm}$ & $10 \mu \mathrm{s}$ & $1.0 \mathrm{AU}$ \\
\hline $\begin{array}{l}\text { Atto } 647 \mathrm{~N}- \\
\text { labeled longevity } \\
\text { testing for } 100 \\
\text { nm beads (oil } \\
\text { and water) }\end{array}$ & $10 \%$ & $50 \%$ & $10 \mathrm{~nm}$ & $10 \mu \mathrm{s}$ & $1.0 \mathrm{AU}$ \\
\hline $\begin{array}{l}\text { Atto 647N- } \\
\text { labeled longevity } \\
\text { testing for } 50 \mathrm{~nm} \\
\text { beads (elevated } \\
\text { temperature, } \\
\text { pitcher plant } \\
\text { fluid, acid, and } \\
\text { soil water) }\end{array}$ & $20 \%$ & $50 \%$ & $5 \mathrm{~nm}$ & $10 \mu \mathrm{s}$ & $1.0 \mathrm{AU}$ \\
\hline $\begin{array}{l}\text { iDye-labeled } \\
\text { plastic longevity } \\
\text { testing (oil and } \\
\text { water) }\end{array}$ & $50 \%$ & $100 \%$ & $10 \mathrm{~nm}$ & $20 \mu \mathrm{s}$ & $1.0 \mathrm{AU}$ \\
\hline $\begin{array}{l}\text { Expanded } \\
\text { polystyrene } \\
\text { debris } \\
\end{array}$ & $40 \%$ & $50 \%$ & $10 \mathrm{~nm}$ & $10 \mu \mathrm{s}$ & $1.0 \mathrm{AU}$ \\
\hline $\begin{array}{l}\text { Polystyrene } \\
\text { sanding debris }\end{array}$ & $5 \%$ & $35 \%$ & $10 \mathrm{~nm}$ & $10 \mu \mathrm{s}$ & $1.0 \mathrm{AU}$ \\
\hline 50 nm PMMA & $10 \%$ & $50 \%$ & $10 \mathrm{~nm}$ & $10 \mu \mathrm{s}$ & $1.0 \mathrm{AU}$ \\
\hline $\begin{array}{l}\text { PTFE } \\
\text { suspension }\end{array}$ & $10 \%$ & $50 \%$ & $10 \mathrm{~nm}$ & $10 \mu \mathrm{s}$ & $1.0 \mathrm{AU}$ \\
\hline C. elegans & $35 \%$ & $75 \%$ & $10 \mathrm{~nm}$ & $10 \mu \mathrm{s}$ & $1.0 \mathrm{AU}$ \\
\hline
\end{tabular}


a

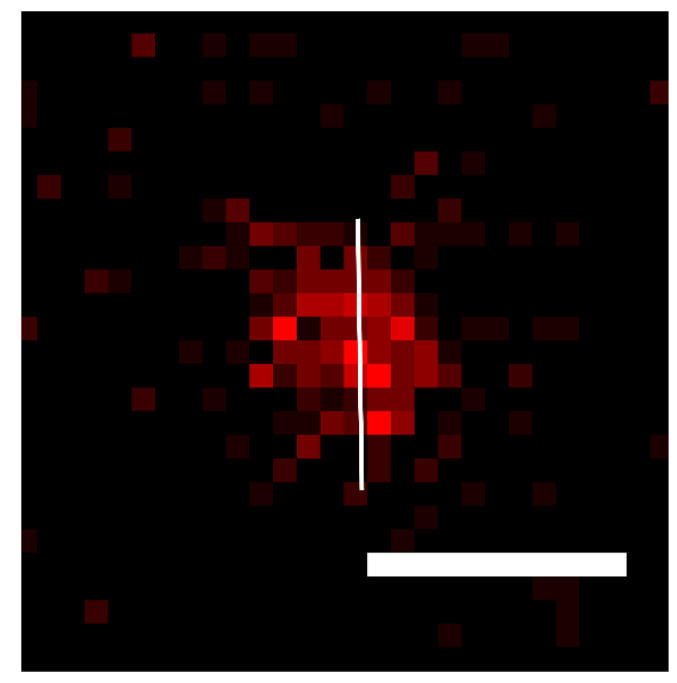

$\mathrm{b}$

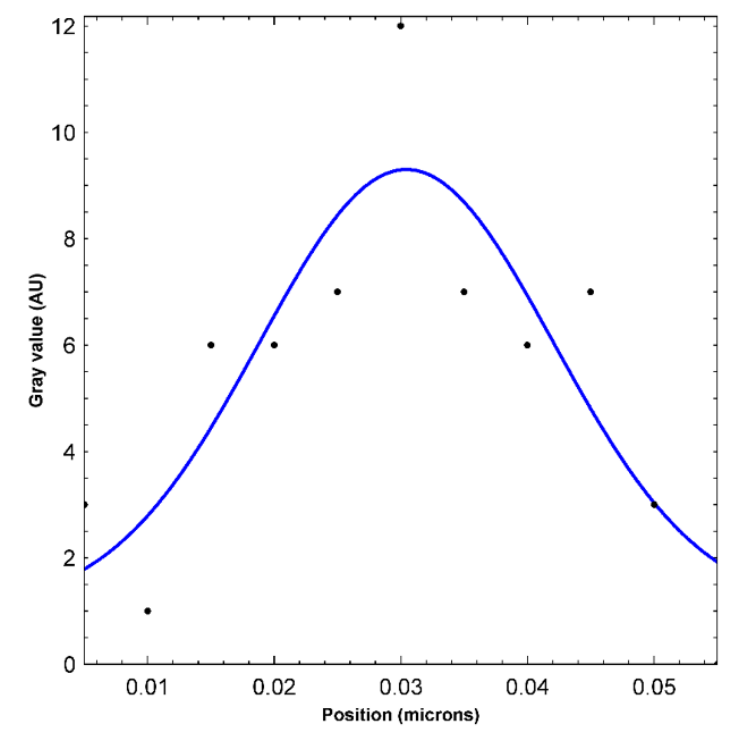

Supplementary Figure 1: Image (a) and (b) point spread function across indicated line (Gaussian fit) of a $50 \mathrm{~nm}$ polystyrene bead passively labeled with Atto $647 \mathrm{~N}$. Scale bar is $50 \mathrm{~nm}$.

a

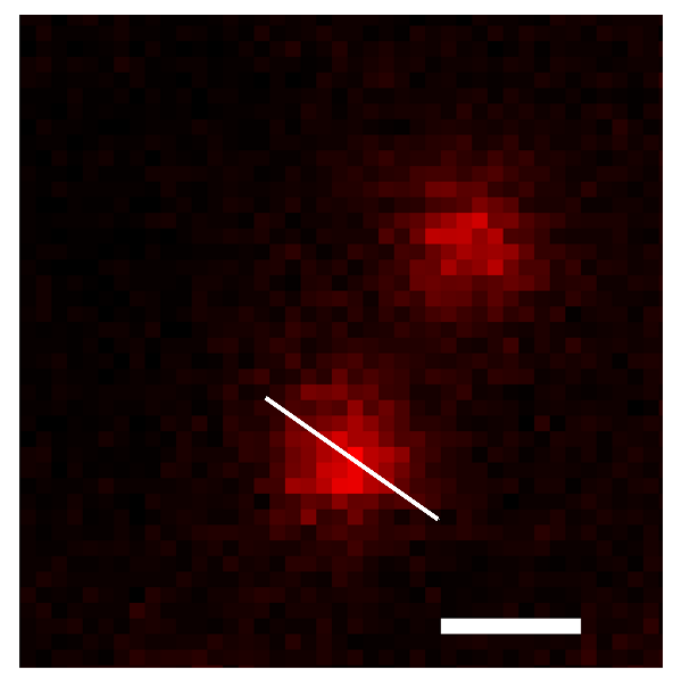

b

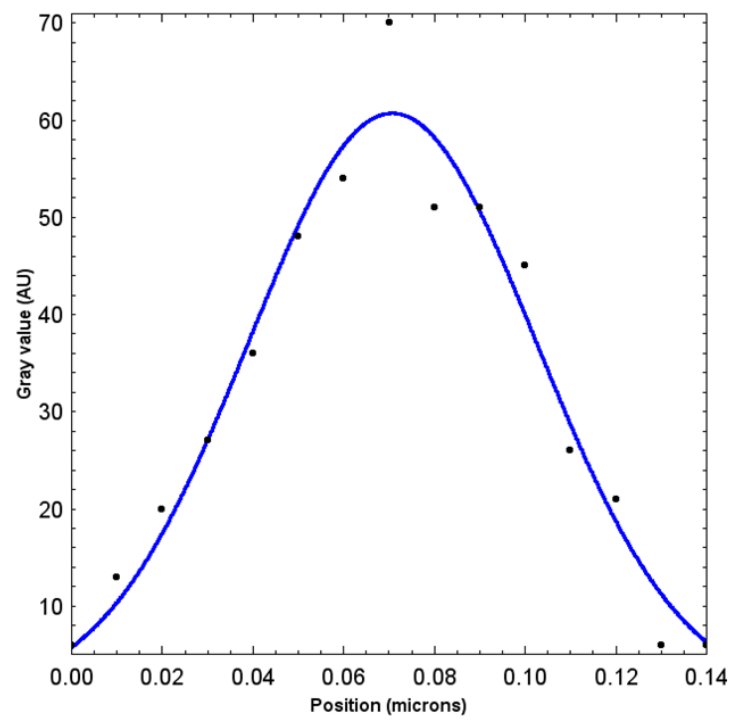

523 Supplementary Figure 2: Image (a) and (b) point spread function across indicated line (Gaussian fit) of a $100 \mathrm{~nm}$ 524 polystyrene bead passively labeled with Atto $647 \mathrm{~N}$. Scale bar is $100 \mathrm{~nm}$. 
a

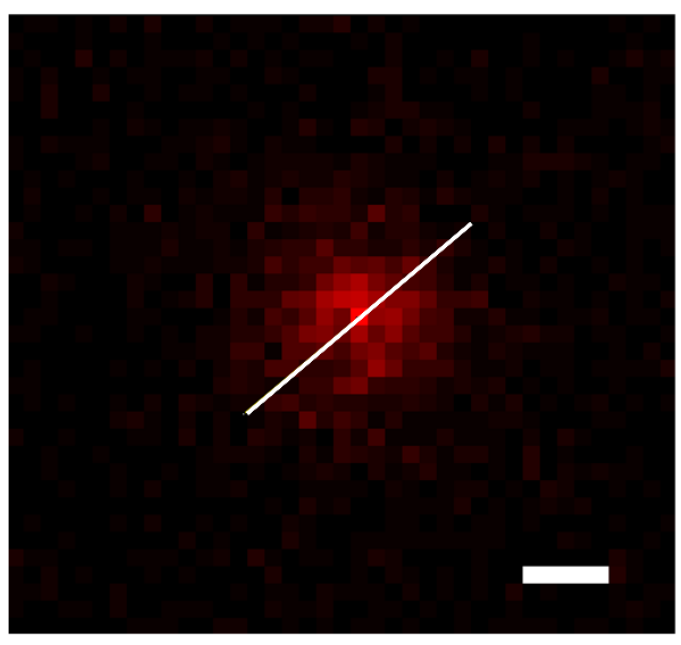

b

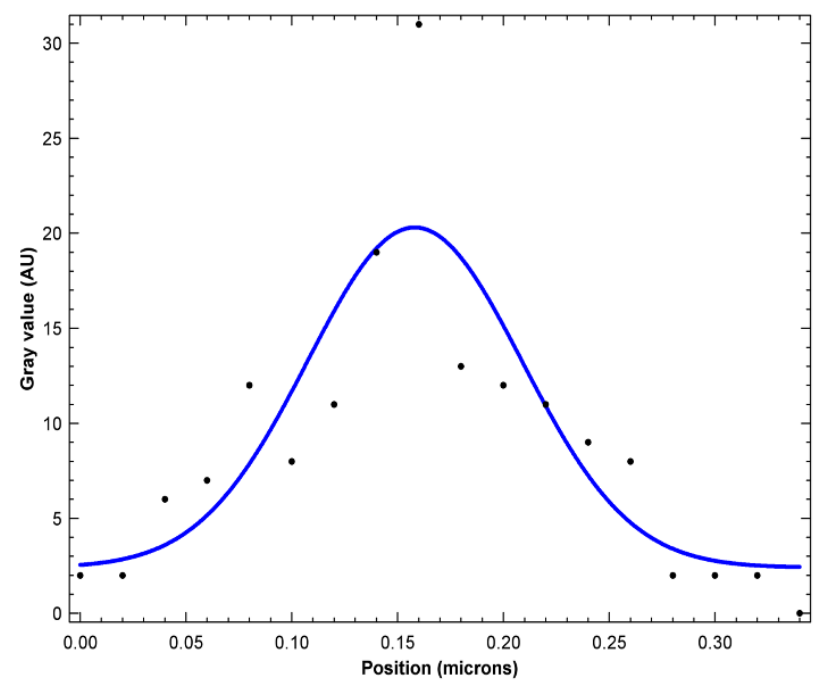

Supplementary Figure 3: Image (a) and (b) point spread function across indicated line (Gaussian fit) of a $100 \mathrm{~nm}$ polystyrene bead swell labeled with iDye Blue. Scale bar is $100 \mathrm{~nm}$.

a

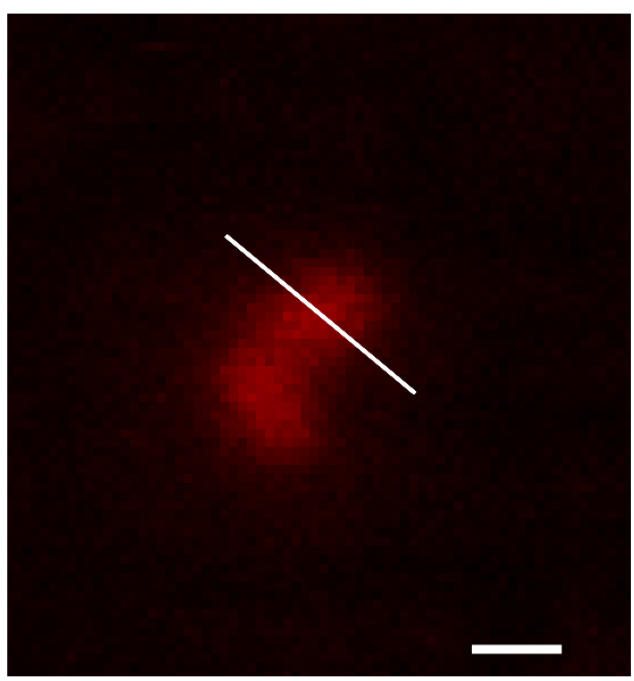

b

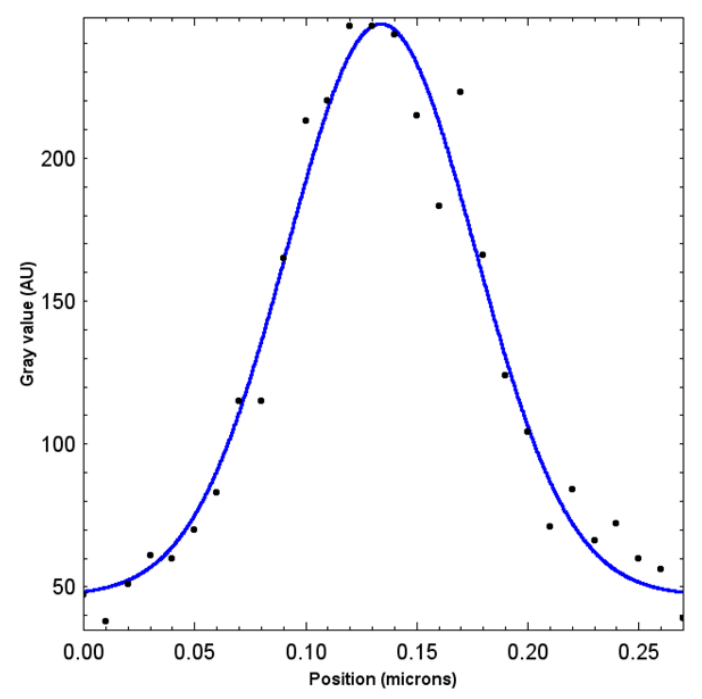

Supplementary Figure 4: Image (a) and (b) point spread function across indicated line (Gaussian fit) of a polystyrene sanding debris passively labeled with Atto 647N. Scale bar is $100 \mathrm{~nm}$. 
bioRxiv preprint doi: https://doi.org/10.1101/2020.08.25.267443; this version posted February 24, 2021. The copyright holder for this preprint (which was not certified by peer review) is the author/funder. All rights reserved. No reuse allowed without permission.

DAY 1

a

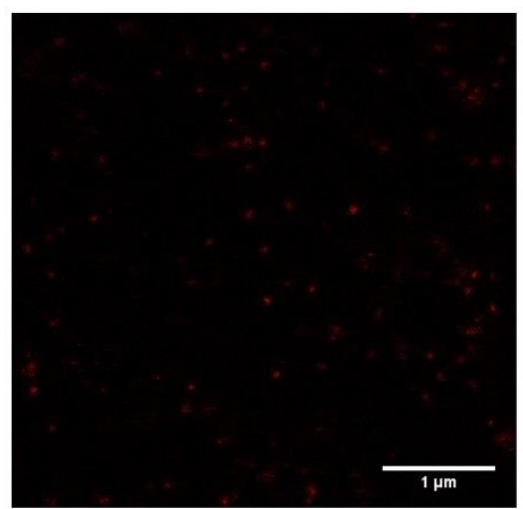

C

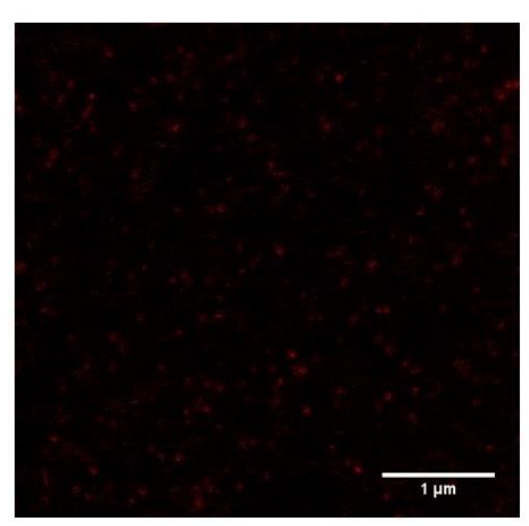

e

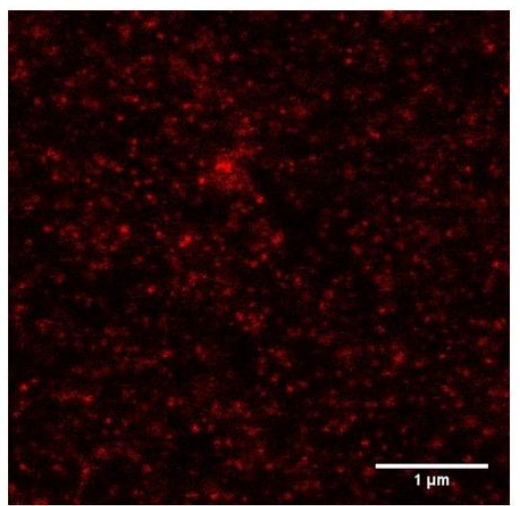

DAY 49

b

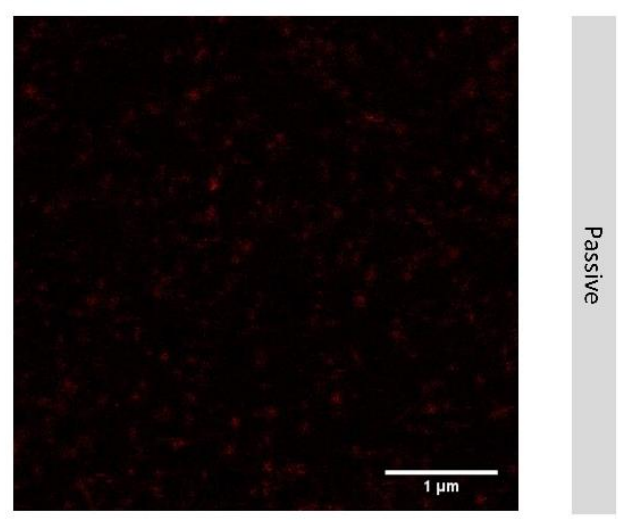

d

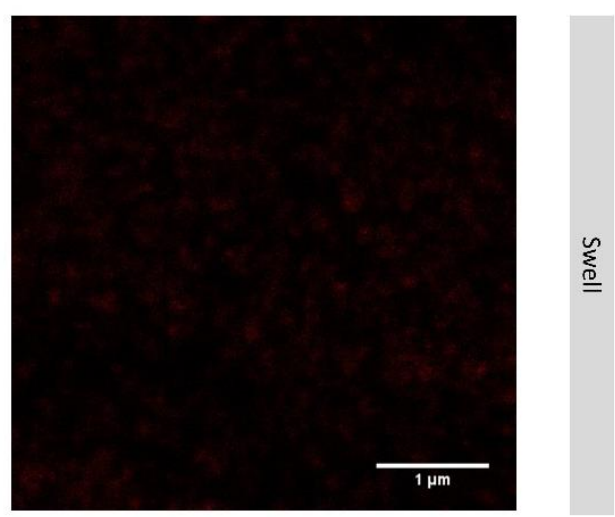

f

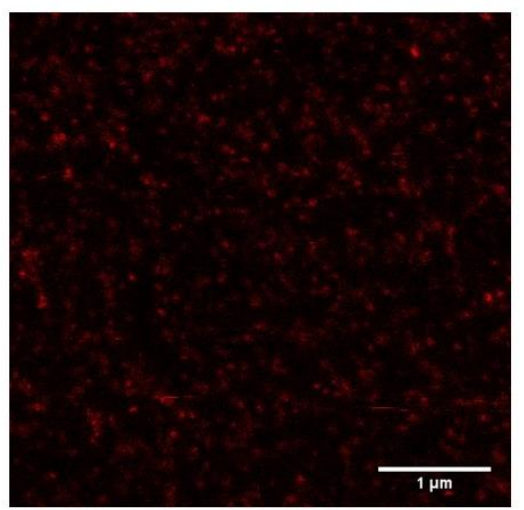

Supplementary Figure 5: Comparison between images of $100 \mathrm{~nm}$ polystyrene beads initially and after 49 days in water for passive $(\mathrm{a}, \mathrm{b})$, swell $(\mathrm{c}, \mathrm{d})$, and covalent $(\mathrm{e}, \mathrm{f})$ labeling with Atto $647 \mathrm{~N}$. 
DAY 1

a

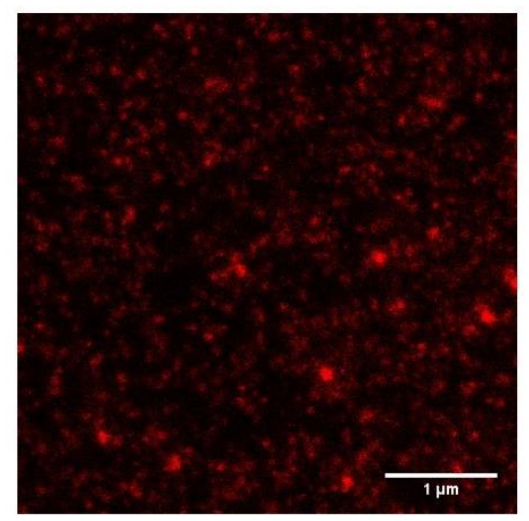

C

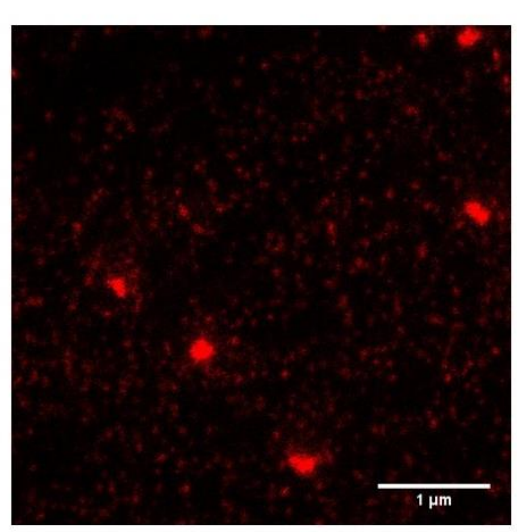

d

e

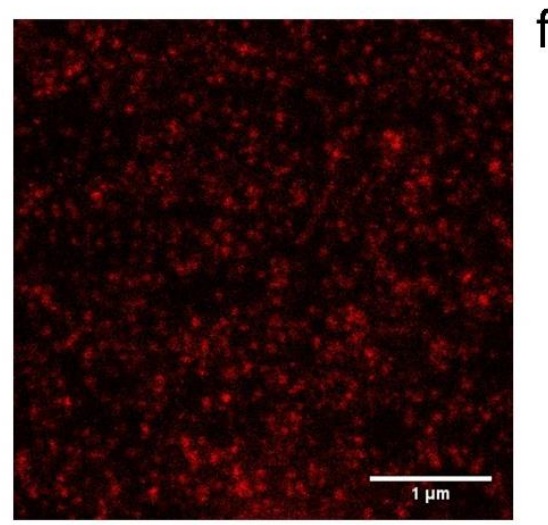

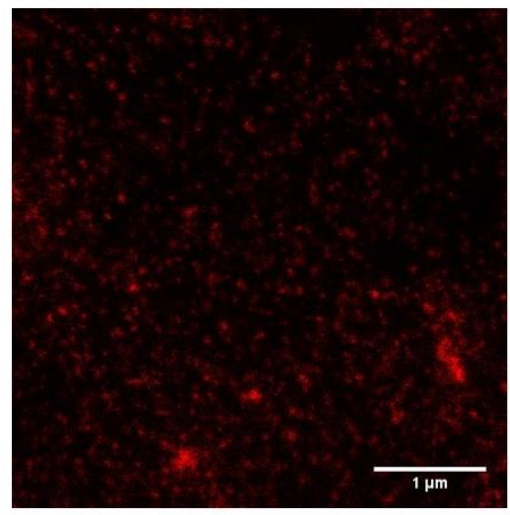

DAY 49
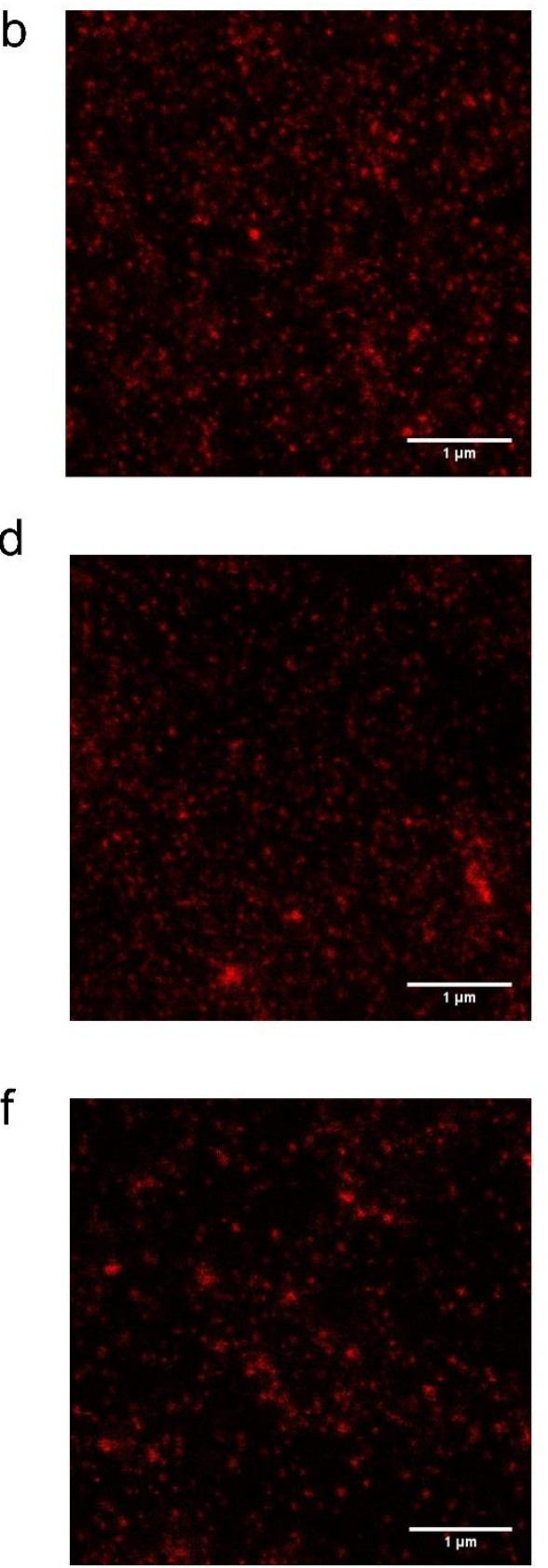
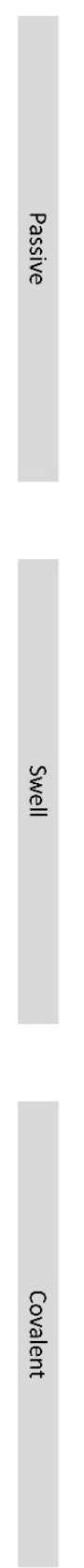

Supplementary Figure 6: Comparison between images of $100 \mathrm{~nm}$ polystyrene beads initially and after 49 days in oil for passive (a,b), swell (c,d), and covalent (e,f) labeling with Atto 647N. 


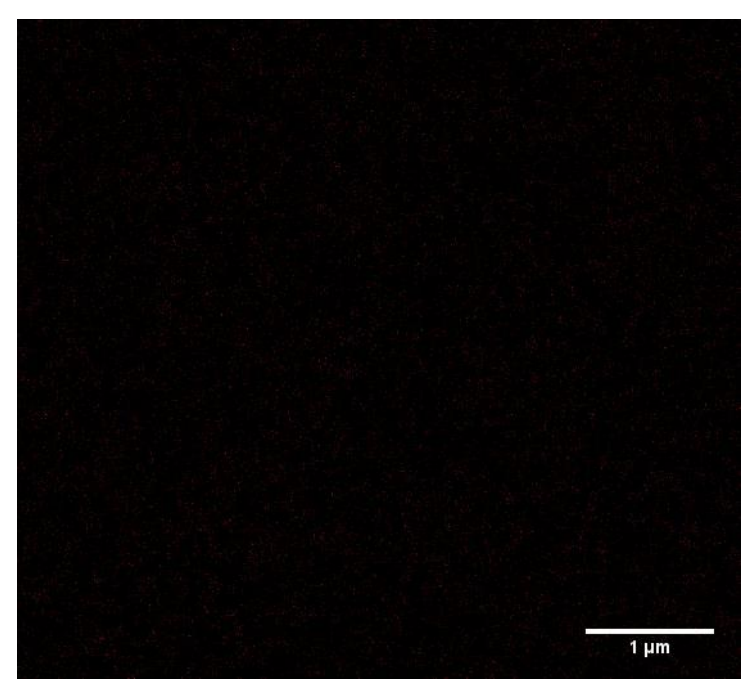

Supplementary Figure 7: Free dye dialyzed control STED image. No detectable signal from dialyzed solution, suggesting that free dye was largely removed with the dialysis step.

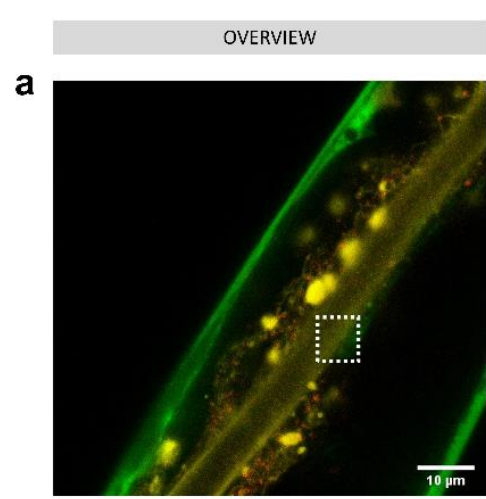

d

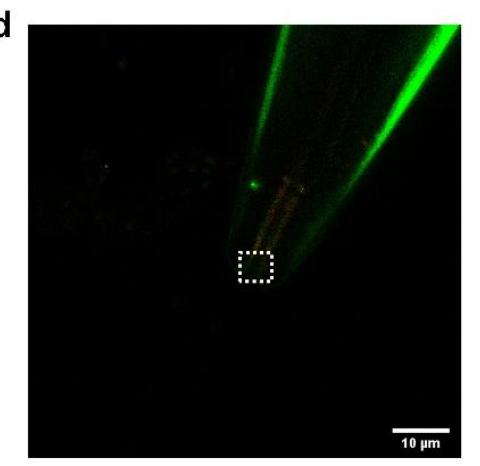

CONFOCAL

b

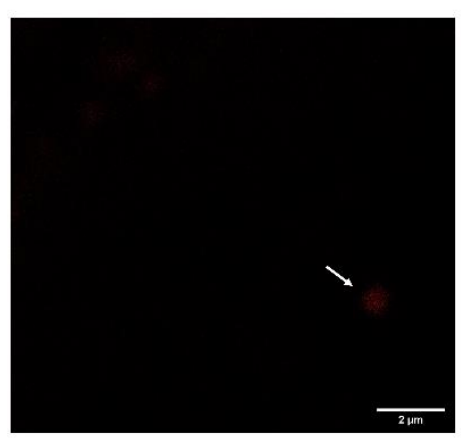

e

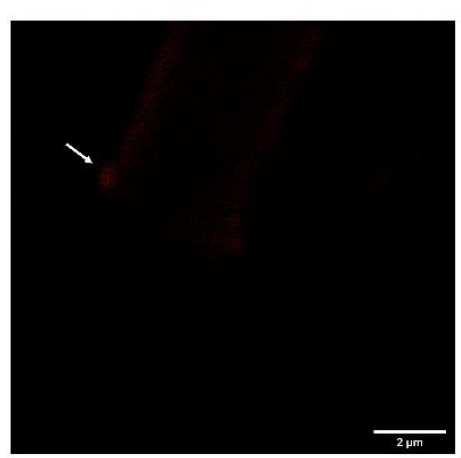

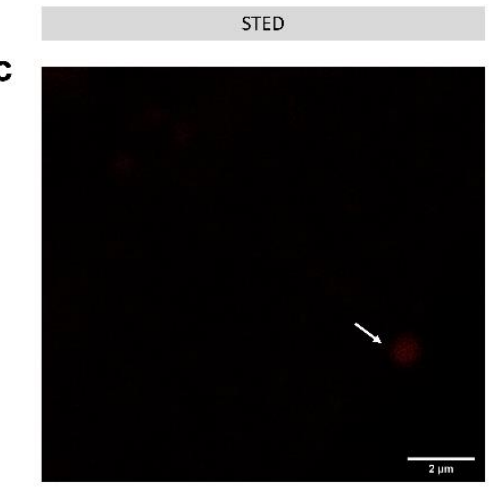

f

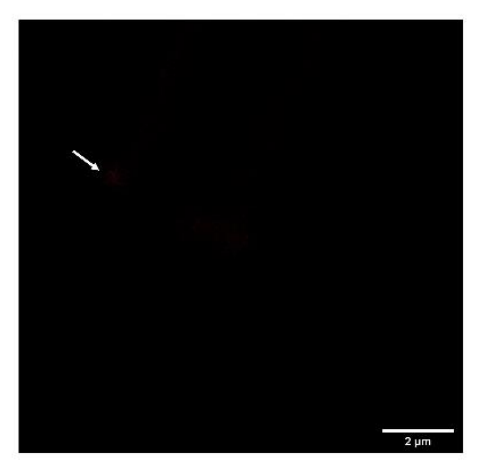

Supplementary Figure 8: Imaging C. elegans KWN117 adult exposed to dialyzed dye solution (no nanoparticles) expressing GFP (green) in the body wall and mCherry (yellow) in the apical intestinal membrane. Confocal overview, and high resolution of confocal and STED images of scanned area indicated by white boxes for parts of the digestive track in the intestine (a,b,c) and mouth $(\mathrm{d}, \mathrm{e}, \mathrm{f})$. Note that while nanoparticles are not seen there is still some signal, likely from autofluorescence, that would have potentially been misinterpreted as labeled nanoplastic particles, due to a truer image size, but for the resolution provided by STED microscopy. Arrows indicate potential false positive signal from confocal imaging where STED imaging reveals that the signal is inconsistent with $50 \mathrm{~nm}$ particles. 
b

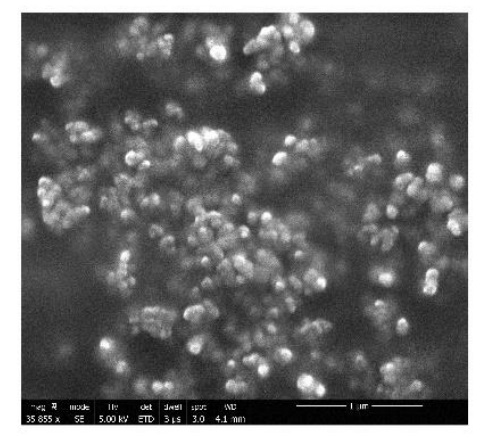

d

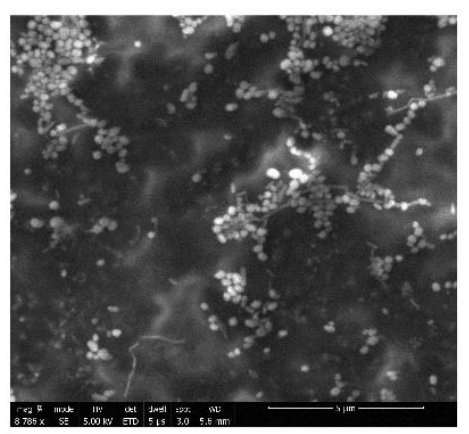

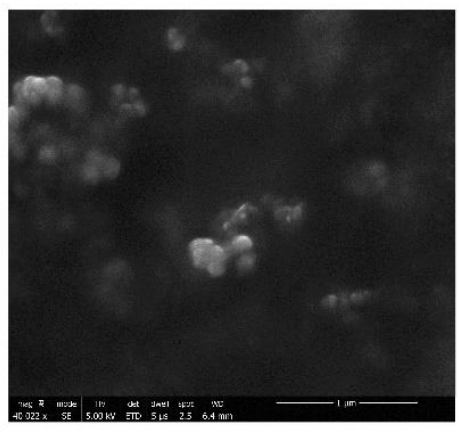

e

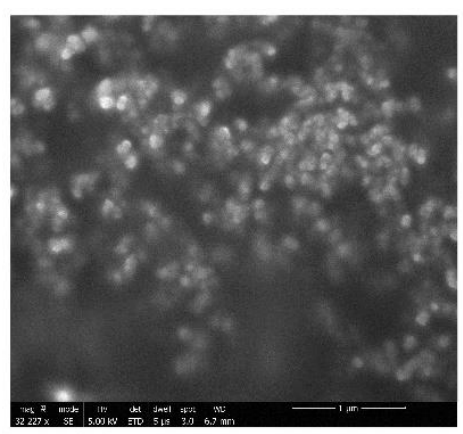

C

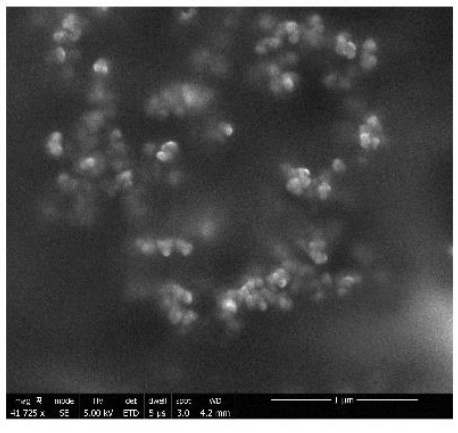

f

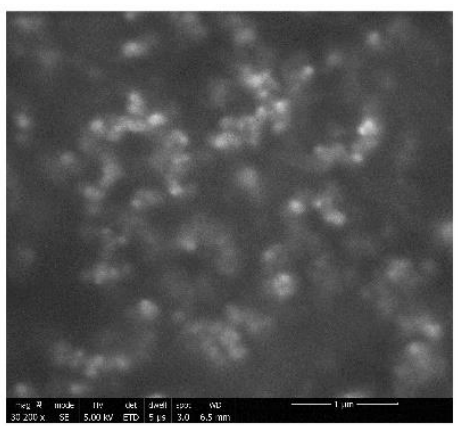

Supplementary Figure 9: SEM images of samples including (a) $100 \mathrm{~nm}$ plain polystyrene beads, (b) $100 \mathrm{~nm}$ aminemodified polystyrene beads, (c) $50 \mathrm{~nm}$ plain polystyrene beads, (d) PTFE particle suspension, (e) debris from a heated polystyrene plate, and (f) debris from sanding a polystyrene petri dish. 